\title{
Voxelated Soft Matter via Multimaterial Multinozzle 3D Printing
}

\section{Citation}

Skylar-Scott, Mark A., Jochen Mueller, Claas W. Visser, and Jennifer A. Lewis. 2019. Voxelated Soft Matter via Multimaterial Multinozzle 3D Printing. Nature 575, no. 7782: 330-35.

\section{Permanent link}

http://nrs.harvard.edu/urn-3:HUL.InstRepos:42083019

\section{Terms of Use}

This article was downloaded from Harvard University's DASH repository, and is made available under the terms and conditions applicable to Other Posted Material, as set forth at http:// nrs.harvard.edu/urn-3:HUL.InstRepos:dash.current.terms-of-use\#LAA

\section{Share Your Story}

The Harvard community has made this article openly available.

Please share how this access benefits you. Submit a story.

\section{Accessibility}




\section{Voxelated Soft Matter via Multimaterial Multinozzle 3D Printing}

Mark A. Skylar-Scott ${ }^{1,2 \dagger}$, Jochen Mueller ${ }^{1,2 \dagger}$, Claas W. Visser ${ }^{1,2}$, and Jennifer A. Lewis ${ }^{1,2,{ }^{*}}$

1. Harvard Paulson School of Engineering and Applied Sciences, Harvard University, Cambridge, Massachusetts 02138, USA

2. Wyss institute for Biologically Inspired Engineering, Harvard University, Cambridge, Massachusetts 02138, USA

* Corresponding author e-mail: jalewis@seas.harvard.edu

$\dagger$ M.A.S.-S. and J.M. contributed equally to this work

Keywords

3D printing, voxelated matter, multinozzle arrays, origami, soft robotics 
There is growing interest in voxelated matter that is rationally designed and fabricated voxel-byvoxel $^{1-4}$. Currently, inkjet-based 3D printing is the only widely adopted method capable of creating 3D voxelated materials with high precision ${ }^{1-4}$, yet the physics of droplet formation requires the use of low-viscosity inks to ensure successful printing 5 . By contrast, direct ink writing (DIW), an extrusion-based 3D printing method, is capable of patterning a far broader range of materials ${ }^{6-13}$. However, it is difficult to generate multimaterial voxelated matter by extruding monolithic cylindrical filaments in a layerwise manner. Here, we report the design and fabrication of voxelated soft matter using multimaterial multinozzle 3D (MM3D) printing, in which their composition, function, and structure are programmed at the voxel scale. Our MM3D printheads exploit the diodelike behavior that arises when multiple viscoelastic materials converge at a junction to enable seamless, high frequency switching between up to eight different materials to create voxels whose volume approaches the nozzle diameter cubed. As exemplars, we fabricated a Miura origami pattern ${ }^{14}$ and a millipede-like, soft robot that locomotes by co-printing multiple epoxy and silicone elastomer inks, respectively, whose stiffness varies by several orders of magnitude. Our method substantially broadens the palette of voxelated materials that can be rationally designed and manufactured in complex motifs.

Physical objects can be broken down and described as a collection of finite volume elements, i.e., voxels, which can be stored as a virtual n-dimensional matrix whose individual elements define the local material composition, structure, and properties. Additive manufacturing (AM) offers the potential to reverse this process by generating voxelated matter from a 3D dataset in which multimaterial building blocks are assembled voxel-by-voxel. AM platforms, augmented by optimization ${ }^{15,16}$ or machine learning tools ${ }^{3,4}$, would afford nearly unlimited design potential. While emerging research is embracing such opportunities, the only AM method well suited for constructing voxelated matter is inkjet-based 3D printing. This method is capable of reproducing exquisitely detailed multimaterial data $\operatorname{sets}^{1-4}$; however, both the number of printable materials and their properties are limited due to the constraints imposed by drop-on-demand printing.

Direct ink writing (DIW) is an alternate 3D printing method that is capable of patterning a broad palette of high performance materials, yet current embodiments rely on extruding cylindrical filaments composed of a single material through a single nozzle in a layerwise manner. An ideal multimaterial DIW system would be able to extrude a multimaterial filament at a sufficiently high switching frequency to create voxel elements ( $\sim D^{3}$ in volume, where $D$ is the nozzle diameter) along its length with specified compositions. While recent advances in the design and implementation of mixing ${ }^{17,18}$ and switching ${ }^{19,20}$ nozzles allow multiple materials to be co-printed continuously, the frequency or resolution at which they 
can switch materials is limited, and thus they are unable to produce such voxelated filaments. Specifically, the large dead volume in mixing nozzles results in long material transitions, while the use of flow-driven syringe pumps to operate switching nozzles limits the switching frequency $(\sim 1 \mathrm{~Hz})$ due to compliance and thread backlash. In addition, print speed constraints that arise from printing 3D objects from single nozzles greatly limit the vast design space that can be realized by voxelated soft matter. To retain part resolution when the linear scale of a $3 \mathrm{D}$ object is increased by a factor of $L$, the number of printed voxels as well as the build time must be increased by $L^{3}$. This scaling behavior is especially prohibitive for manufacturing large parts using reactive ${ }^{17}$ or biological ${ }^{21}$ inks with short printability windows. To reduce build times, 1D multinozzle arrays have been developed that split a single inlet stream of ink into multiple continuous ink filaments that exit simultaneously from the printhead ${ }^{22}$. However, since each nozzle is only able to extrude a single material, the $3 \mathrm{D}$ objects produced via these multinozzle arrays are limited in complexity.

Here, we report a multimaterial multinozzle 3D (MM3D) printing method for generating voxelated soft matter that integrates ultra-rapid multimaterial switching for enhanced complexity with multinozzle printing for enhanced build speed (Fig. 1a). Using MM3D printing, we produced complex architectures composed of multiple materials with controlled composition, function, and structure in a voxel-by-voxel manner (Fig. 1a). We designed and rapidly fabricated MM3D printheads in the form of OD (single nozzle), 1D, and 2D nozzle arrays using stereolithography (Fig. 1b, c, Extended Data Fig. 1a-c). These MM3D printheads are capable of depositing up to eight materials, each of which flows through independent bifurcating channel networks that reside within the printhead before merging into a single ink flow immediately prior to the nozzle outlet (Extended Data Fig. 1d,e). To ensure uniform printing, each nozzle within the printhead must possess inner diameters with a coefficient of variation below $\sim 5 \%$ (Extended Data Fig. 2) ${ }^{22}$. Each printhead is connected to multiple syringes, each of which contains a different material, that are actuated by a fast-cycling bank of pneumatic solenoids to enable high-frequency switching (Fig. 1d). Using this embodiment, 3D objects must be produced in periodic layouts, since each nozzle is not independently switchable. Importantly, MM3D printheads are capable of generating voxelated matter at characteristic build times that scale with $L$ rather than $L^{3}$ for single nozzles (Fig. 1e, Supplementary Video 1, Extended Data Fig. 3, and Methods for a further analysis).

Modeling multimaterial switching. A salient feature of our MM3D printheads is their $S_{\text {ink }}$-to-1 junctions, where multiple material channels converge into a single outlet to generate continuous, yet heterogeneous, filaments whose composition and properties are specified at the voxel level (Fig. 2a). For MM3D printing, we opted to use pressure-driven flows to enable faster start-stop dynamics (i.e., higher switching frequencies) compared to the syringe pump-driven flows used previously ${ }^{19}$. However, pressuredriven extrusion requires careful optimization of ink rheology, nozzle geometry, and printing pressure to 
prevent ink from one channel flowing upstream into a neighboring channel that houses a different ink. If backflow occurs, then the wrong voxel composition would be extruded immediately after switching. To model and optimize this parameter space, we created Y-junction nozzles and directly observed ink flows (Fig. 2b). For Newtonian inks, the equivalent circuit would be a resistive network, wherein any material flow on one side would drive a backflow in the other channel, rendering MM3D printing impossible (Fig. 2c). By contrast, two viscoelastic inks, i.e., Herschel-Bulkley fluids with shear yield stresses, exhibit nonlinear flow behavior similar to a network of transient voltage suppressor (TVS) diodes. Here, a finite flow $Q_{f}<Q_{f}^{\max }$ in one channel can occur while maintaining a static condition on the other channel, i.e., $Q_{s}=$ 0 . This zero-backflow condition persists so long as the back pressure generated at the junction, $p_{j}$, does not exceed a critical value, $p_{c}$, that would result in a yielding of the static material (Fig. 2d, Supplementary Video 2, Extended Data. Fig. 4). We derived an analytical model for $p_{c r}$ and found that the corresponding maximum ink flow rate $Q_{f}^{\max }$ depends on channel geometry and ink rheology (see Methods for an extended discussion). To test this model, we flowed a range of silicone inks of varying viscosity and shear yield stress values and experimentally detected the yielding of the static ink to quantify values of $Q_{f}^{\max }$ (Fig. 2e, Extended Data Fig. 5). As predicted, $Q_{f}^{\max }$ and, hence, the maximum print speed, are dependent upon the shear yield stress of the static ink and the apparent viscosity of the flowing ink (Fig. 2f). The geometry of the nozzle also significantly affects $Q_{f}^{\max }$; longer inlet (Fig. 2g) or shorter outlet (Fig. 2h) channels enable higher print speeds. The use of asymmetric MM3D printheads facilitates printing of multiple inks with widely varying rheological behavior. For example, asymmetric printheads can be designed with longer channels for the lower viscosity ink, such that the maximum print speed for each ink is better matched to enable constant printhead movement (Fig. 2i).

MM3D printing performance. To generate continuous multimaterial filaments composed of voxelated elements, we operate the MM3D printheads at a switching frequency of up to $50 \mathrm{~Hz}$ (Fig. 3a, Supplementary Video 3 ) that enable 3D objects to be rapidly printed in a voxel-based manner. For example, using $8 \times 1$ 1D MM3D printheads, voxelated soft matter can be generated by scanning either 'parallel' lines, or by completing each 'island' of material before switching to the next (Fig. 3b, c, Extended Data Fig. 6). The parallel method enables simpler print path design with fewer aberrations arising due to filament cornering compared to the island method. However, the island method enables fewer material transitions and therefore can generate sharper borders. When co-printing representative silicone and wax inks at high speeds, the 'more viscous' silicone ink had a propensity to backflow into the 'less viscous' wax channel (i.e. $Q_{f \mid \text { silicone }}^{\max }<Q_{f \mid \text { wax }}^{\max }$ ), causing backflow aberrations (Extended Data Fig. 7). To mitigate this, we designed a matched asymmetric nozzle with a narrower channel for delivering the wax ink. A 0D asymmetric nozzle could raster arbitrary high-fidelity patterns with a single continuous filament with a 
diameter of $250 \mu \mathrm{m}$ (Supplementary Video 4). At material switch points, the $90-10 \%$ transition is measured at $\sim 320 \mu \mathrm{m}$, which, at a print speed of $20 \mathrm{~mm} / \mathrm{s}$, represents a switch time of $16 \mathrm{~ms}$ and a characteristic switching frequency of $\sim 60 \mathrm{~Hz}$. Using data derived from a bitmap, large, periodic, and complex patterns were rapidly produced via a parallel print path method using an $8 \times 1$ two-material printhead via MM3D printing (Fig. 3d, e, Supplementary Video 5). To demonstrate the island printing method, we created continuous meandering shapes using an $8 \times 1$ four-material MM3D printhead (Fig. 3f, g, Supplementary Video 6). We note that MM3D printheads are capable of patterning a broad range of structural, functional and biological inks developed for DIW, including gelatin, which exhibits the requisite yielding behavior for bioprinting ${ }^{12,20,21,23}$ (Extended Data Fig. 8).

MM3D printing of functional objects. To demonstrate the ability to spatially program the composition, structure and properties of printed objects, we first fabricated a Miura origami pattern (Fig. 4a, b, Supplementary Video 7) using an $8 \times 1$ 1D MM3D printhead. This pattern, which is traditionally produced by folding paper, allows one to transform a sheet with a large surface area into a compact 3D structure $^{14,24}$. Others have used laser cutting to generate multiple stacked layers, which are then manually assembled to create flexible and stiff regions ${ }^{25-27}$. Using MM3D printing, we generated Miura patterns rapidly in a single step by co-depositing two viscoelastic epoxy inks which, when cured, exhibit different Young's moduli: $0.61 \pm 0.16 \mathrm{MPa}$ for the flexible hinges and 3.92 $\pm 0.40 \mathrm{GPa}$ for the stiff origami faces (Fig. $4 \mathrm{c}, \mathrm{d}$ ). These hinges display a characteristic transition, from soft to stiff, over a distance of $\sim 400 \mu \mathrm{m}$ (Fig. 4e). Since reactive epoxy inks typically have short pot-lives (Extended Data Fig. 9a-d), MM3D printing is well suited for fabricating these large-scale structures due to the greatly reduced build times needed (Fig. 4f). The resulting Miura structure exhibits high deformability in the hinges and is capable of multiple folding cycles (1632 \pm 217 ) before failing (Supplementary Video 7, Extended Data Figs. 9e-g). Notably, failure typically occurs within the flexible hinge, away from the material transition zone.

To further highlight the capabilities of MM3D printing, we designed and fabricated a soft robot composed of rigid and soft elastomers patterned with millipede-like features and embedded pneumatic actuators in modular form (Fig. 5a, Extended Data Fig. 10, Supplementary Video 8). Many soft robots rely on actuators arranged in periodic geometries, making them well suited to MM3D printing ${ }^{28-29}$. In our soft robotic walkers, each millipede walker module is composed of 16 pneumatic actuators that are interconnected by a pair of underlying pneumatic networks with one channel that connects the inner two columns of actuators and another channel that connects the outer two columns. When a vacuum is applied to these channels, the soft silicone 'muscle' buckles, causing a lateral and vertical displacement of the stiff 'leg' ( $\Delta x$ and $\Delta y$, respectively) (Fig. 5b). To realize this design, we simultaneously printed both flexible $(0.225 \pm 0.005 \mathrm{MPa}$, blue) and stiff $(1.81 \pm 0.05 \mathrm{MPa}$, grey) silicone inks (Fig. $5 \mathrm{c})$ in a motif that includes 
both overhanging walls and spanning gaps that generate closed channels. To achieve a walking motion, the legs are actuated as shown in Fig. $5 \mathrm{~d}$, e, such that the $\Delta y$ displacement results in the weight of the soft robot resting on alternating actuator groups. The anti-symmetric arrangement of the two sets of actuators ensures that the power strokes for each actuator group provide a $\Delta x$ lateral displacement in a consistent direction to provide forward locomotion. At its optimum cycling frequency of $5 \mathrm{~Hz}$, the soft robotic actuators (weighing $\sim 25 \mathrm{~g}$ ) could move at $\sim 1 \mathrm{~cm} / \mathrm{s}$ while carrying loads of up to $\sim 200$ g, i.e., 8 times their mass (Fig. $5 \mathrm{f}, \mathrm{g}$, Supplementary Video 9). Finally, two or more of these actuator modules can be connected in series to produce longer, millipede-like soft robots that carry more load (Fig. 5h, Supplementary Video 10).

Summary and conclusions. We present a powerful and versatile approach for generating voxelated soft matter based on MM3D printing. By harnessing the pressure-driven flow behavior of yieldstress fluids, we seamlessly switch between multiple materials at high frequencies to enable continuous printing of heterogenous voxelated filaments. By designing and implementing multimaterial multinozzle printheads, we rapidly constructed 3D objects with spatially programmed composition, structure, and properties. While two embodiments, namely foldable origami patterns and soft robots that locomote, were printed to highlight these capabilities, a broad range of high-performance structural, functional, and biological materials can be patterned by MM3D printing. Moving forward, the next-generation of MM3D printheads would ideally feature independently addressable nozzles with smaller diameters arranged in even larger arrays. These improvements would eliminate the periodicity constraints imposed by the current printhead design, while simultaneously leading to improved feature resolution and lower build times. Ultimately, we envision creating $3 \mathrm{D}$ voxelated matter on demand with superlative performance via the close coupling of MM3D printing, predictive design and optimization.

\section{References}

1. Hosny, A. et al. From improved diagnostics to presurgical planning: High-resolution functionally graded multimaterial 3D printing of biomedical tomographic data sets. 3D Print. Addit. Manuf. 5, 103-113 (2018).

2. Bader, C. et al. Making data matter: Voxel printing for the digital fabrication of data across scales and domains. Sci. Adv. 4, eaas8652 (2018).

3. Gu, G. X., Chen, C. T., Richmond, D. J. \& Buehler, M. J. Bioinspired hierarchical composite design using machine learning: Simulation, additive manufacturing, and experiment. Mater. Horizons 5, 939-945 (2018).

4. Gu, G. X., Chen, C. T. \& Buehler, M. J. De novo composite design based on machine learning 
algorithm. Extrem. Mech. Lett. 18, 19-28 (2018).

5. Jang, D., Kim, D. \& Moon, J. Influence of fluid physical properties on ink-jet printability. Langmuir 25, 2629-2635 (2009).

6. Truby, R. L. \& Lewis, J. A. Printing soft matter in three dimensions. Nature 540, 371-378 (2016).

7. Raney, J. R. et al. Rotational 3D printing of damage-tolerant composites with programmable mechanics. Proc. Natl. Acad. Sci. 115, 1198-1203 (2018).

8. Wehner, M. et al. An integrated design and fabrication strategy for entirely soft, autonomous robots. Nature 536, 451 (2016).

9. Valentine, A. D. et al. Hybrid 3D Printing of Soft Electronics. Adv. Mater. 29, 1703817 (2017).

10. Kotikian, A., Truby, R. L., Boley, J. W., White, T. J. \& Lewis, J. A. 3D Printing of Liquid Crystal Elastomeric Actuators with Spatially Programed Nematic Order. Adv. Mater. 30, 1706164 (2018).

11. Kong, Y. L. et al. 3D printed quantum dot light-emitting diodes. Nano Lett. 14, 7017-7023 (2014).

12. Kolesky, D. B., Homan, K. A., Skylar-Scott, M. A. \& Lewis, J. A. Three-dimensional bioprinting of thick vascularized tissues. Proc. Natl. Acad. Sci. 113, 3179-3184 (2016).

13. Compton, B. G. \& Lewis, J. A. 3D-printing of lightweight cellular composites. Adv. Mater. 26, 5930-5935 (2014).

14. Schenk, M. \& Guest, S. D. Geometry of Miura-folded metamaterials. Proc. Natl. Acad. Sci. 110, 3276-3281 (2013).

15. Bendsøe, M. P. \& Sigmund, O. Material interpolation schemes in topology optimization. Arch. Appl. Mech. 69, 635-654 (1999).

16. Haftka, R. T. \& Gürdal., Z. Elements of Structural Optimization. (Springer Science \& Business Media, 2012).

17. Ober, T. J., Foresti, D. \& Lewis, J. A. Active mixing of complex fluids at the microscale. Proc. Natl. Acad. Sci. U. S. A. 112, 12293-8 (2015).

18. Kokkinis, D., Bouville, F. \& Studart, A. R. 3D Printing of Materials with Tunable Failure via Bioinspired Mechanical Gradients. Adv. Mater. 30, 1705808 (2018).

19. Hardin, J. O., Ober, T. J., Valentine, A. D. \& Lewis, J. A. Microfluidic printheads for multimaterial 3D printing of viscoelastic inks. Adv. Mater. 27, 3279-3284 (2015). 
20. Liu, W. et al. Rapid Continuous Multimaterial Extrusion Bioprinting. Adv. Mater. 29, 1604630 (2017).

21. Kolesky, D. B. et al. 3D bioprinting of vascularized, heterogeneous cell-laden tissue constructs. Adv. Mater. 26, 3124-3130 (2014).

22. Hansen, C. J. et al. High-Throughput Printing via Microvascular Multinozzle Arrays. Adv. Mater. 25, 96-102 (2013).

23. Skylar-Scott, M. A. et al. Biomanufacturing of organ-specific tissues with high cellular density and embedded vascular channels. Sci. Adv. 5, eaaw2459 (2019).

24. Miura, K. The science of Miura-ori: A review. in 4th International Meeting of Origami Science, Mathematics, and Education, RJ Lang, ed., AK Peters, Natick, MA 87-100 (2009).

25. Overvelde, J. T. B., Weaver, J. C., Hoberman, C. \& Bertoldi, K. Rational design of reconfigurable prismatic architected materials. Nature 541, 347 (2017).

26. Babaee, S., Overvelde, J. T. B., Chen, E. R., Tournat, V. \& Bertoldi, K. Reconfigurable origamiinspired acoustic waveguides. Sci. Adv. 2, e1601019 (2016).

27. Boatti, E., Vasios, N. \& Bertoldi, K. Origami metamaterials for tunable thermal expansion. Adv. Mater. 29, (2017).

28. Bartlett, N. W. et al. A 3D-printed, functionally graded soft robot powered by combustion. Science 349, 161-5 (2015).

29. Polygerinos, P. et al. Soft Robotics: Review of Fluid-Driven Intrinsically Soft Devices; Manufacturing, Sensing, Control, and Applications in Human-Robot Interaction. Adv. Eng. Mater. 19, 1700016 (2017). 


\section{Acknowledgements}

The authors thank Prof. Katia Bertoldi for helpful discussions on origami. We also thank Lansing Sugita for her experimental assistance as well as James C. Weaver and Lori K. Sanders for their assistance with imaging, photography, and videography, and Travis Busbee for silicone ink formulations. This work was supported by the Vannevar Bush Faculty Fellowship Program sponsored by the Basic Research Office of the Assistant Secretary of Defense for Research and Engineering through the Office of Naval Research Grant N00014-16-1-2823, the Harvard MRSEC (Grant No. DMR-1420570), and by a generous donation from the GETTYLAB.

\section{Contribution Statement}

M.A.S.-S., J.M., and J.A.L. conceived the project. M.A.S.-S. and J.M. designed experiments. M.A.S.-S., J.M., and C.V. conducted experiments and analyzed data. M.A.S.-S., J.M., and J.A.L. prepared the manuscript.

\section{Data Availability}

The datasets generated during and/or analyzed during the current study are available from the corresponding author on reasonable request.

\section{Code availability}

The code generated during the current study is available from the corresponding author on reasonable request.

\section{Reprints}

Reprints and permissions information is available at www.nature.com/reprints.

\section{Competing Interests}

Jennifer A. Lewis is a co-founder of Voxe18, Inc. and Mark Skylar-Scott owns stock in Formlabs. A patent has been filed by Harvard University on this research.

\section{Correspondence}

Correspondence and requests for materials should be addressed to jalewis@seas.harvard.edu. 


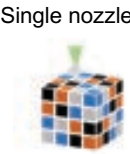

1D multinozzle array

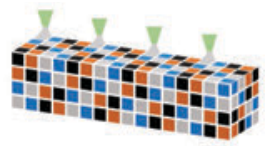

2D multinozzle array

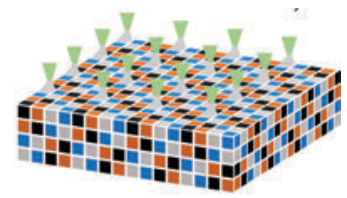

b

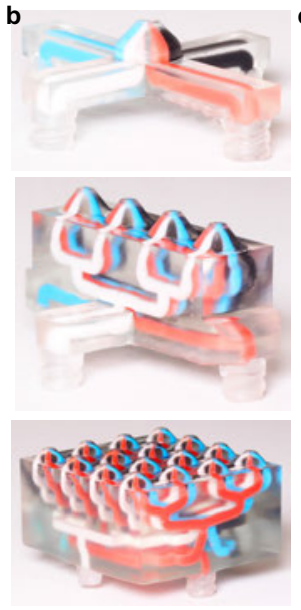

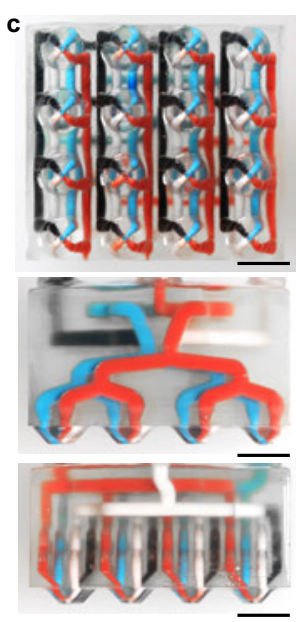

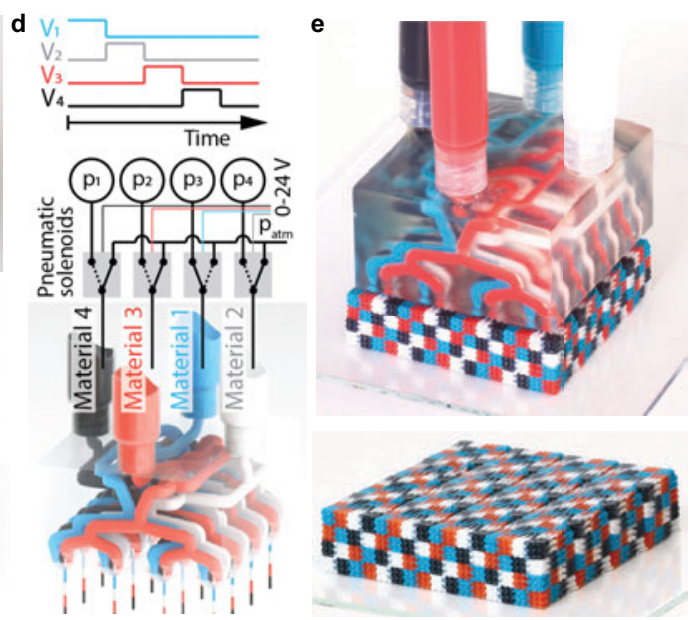

Figure 1 | Multimaterial multinozzle 3D printheads. a, A schematic of voxelated architectures printed using a single (0D) nozzle as well as the 1D and 2D MM3D printheads. b, Photographs of corresponding 0D, 1D, and 2D four-material MM3D printheads. c, Photographs showing top and side views of a 4 $\times 4$ nozzle, four-material, 2D MM3D printhead. Scale bars $=10 \mathrm{~mm}$. d, Schematic depiction of MM3D printhead operation. e, Voxelated matter produced by MM3D printing using a $4 \times 4$-nozzle, four-material, $2 \mathrm{D}$ printhead. 
a

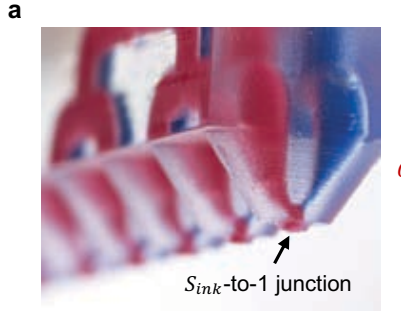

b

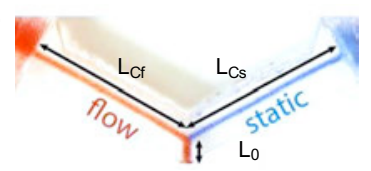

Syringe pump $\left(Q_{f}\right) \quad$ Flow meter $\left(Q_{S}\right)$
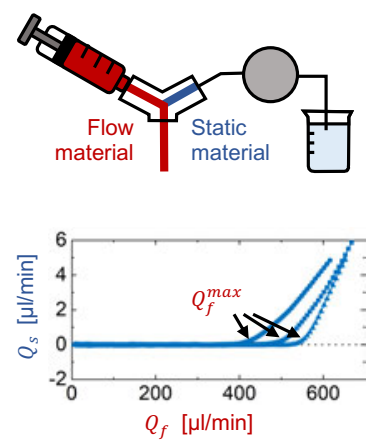
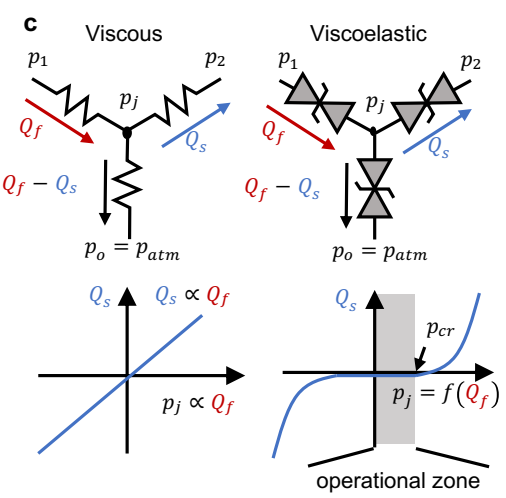

f

$$
\text { 들 }
$$

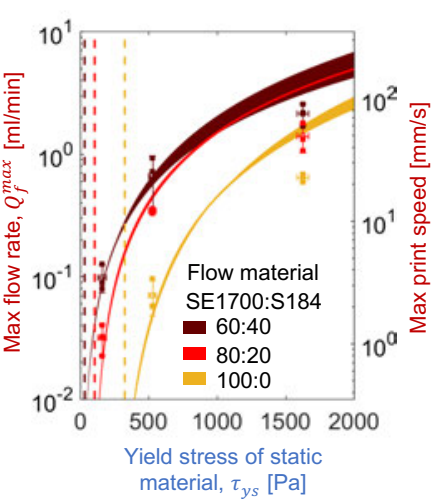

g d
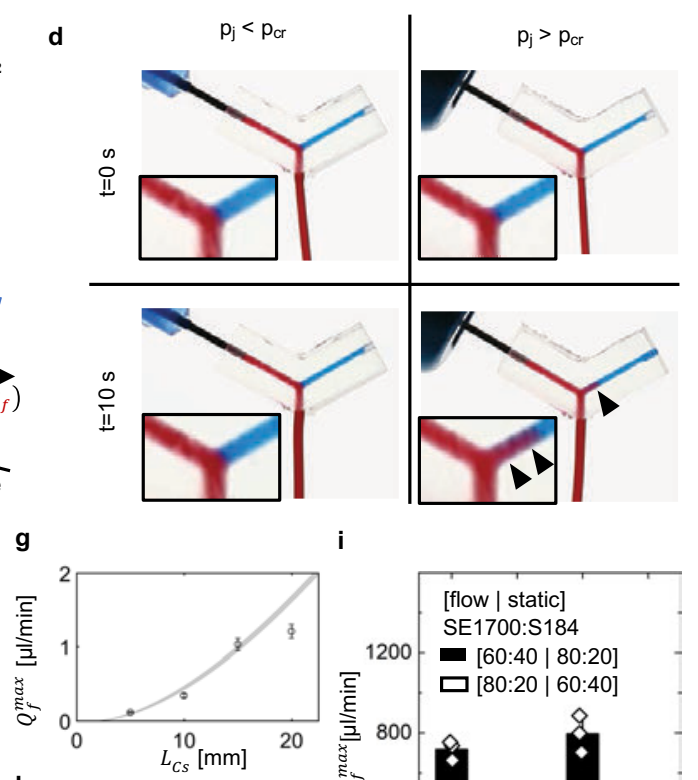

h

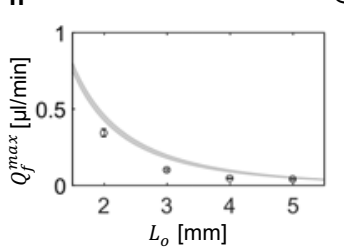

i

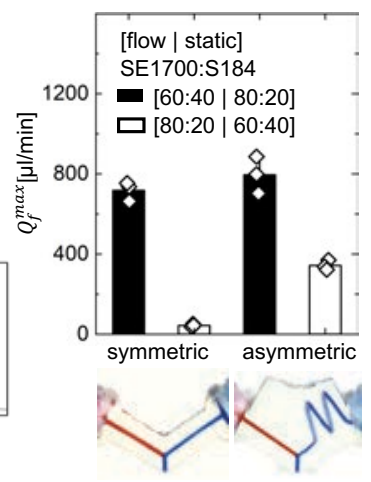

Figure 2 | Viscoelastic ink flow through MM3D printheads. a, An $8 \times 1$ two-material MM3D printhead

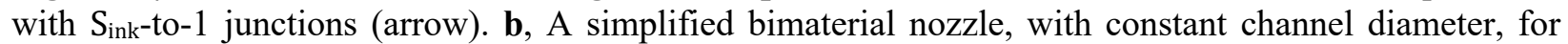
modeling viscoelastic flow behavior. c, Analogous circuit diagrams for viscous (left) and viscoelastic yieldstress (right) materials at a Y-junction, and illustrative plots for $Q_{s}$ vs $p_{j}$. d, Time-lapse of viscoelastic flow in a Y-junction at low (left) and high (right) extrusion rates. Arrowheads indicate the occurrence of backflow. e, Schematic showing the setup used for measuring the yielding of the static material under a ramping extrusion rate of the flow material, and representative plot obtained for $Q_{s}$ vs $Q_{f}$. f, Maximum flow rate, $Q_{f} \max$ for various flow materials when printed alongside various static materials. Shaded curves represent the maximum and minimum bounds of the predicted values, from Eq. 14, from $n=3$ measurements of flow curves for each flow material. Horizontal error bars denote the $95 \%$ confidence interval for the value of the yield stress extracted from Extended Data Fig. 4c. Vertical error bars denote the standard deviation of the flow measurements from $n=3$ experiments using separate nozzles. Dashed lines represent zeros from Eq. 14. g, $Q_{f} \max$ increases with the length of the static material channel and $\mathbf{h}$, decreases with the length of the outlet channel. i, $Q_{f} \max$ for two different inks in matched and unmatched nozzles. Error bars in g-i represent the standard deviation from $n=3$ experiments using separate nozzles. 

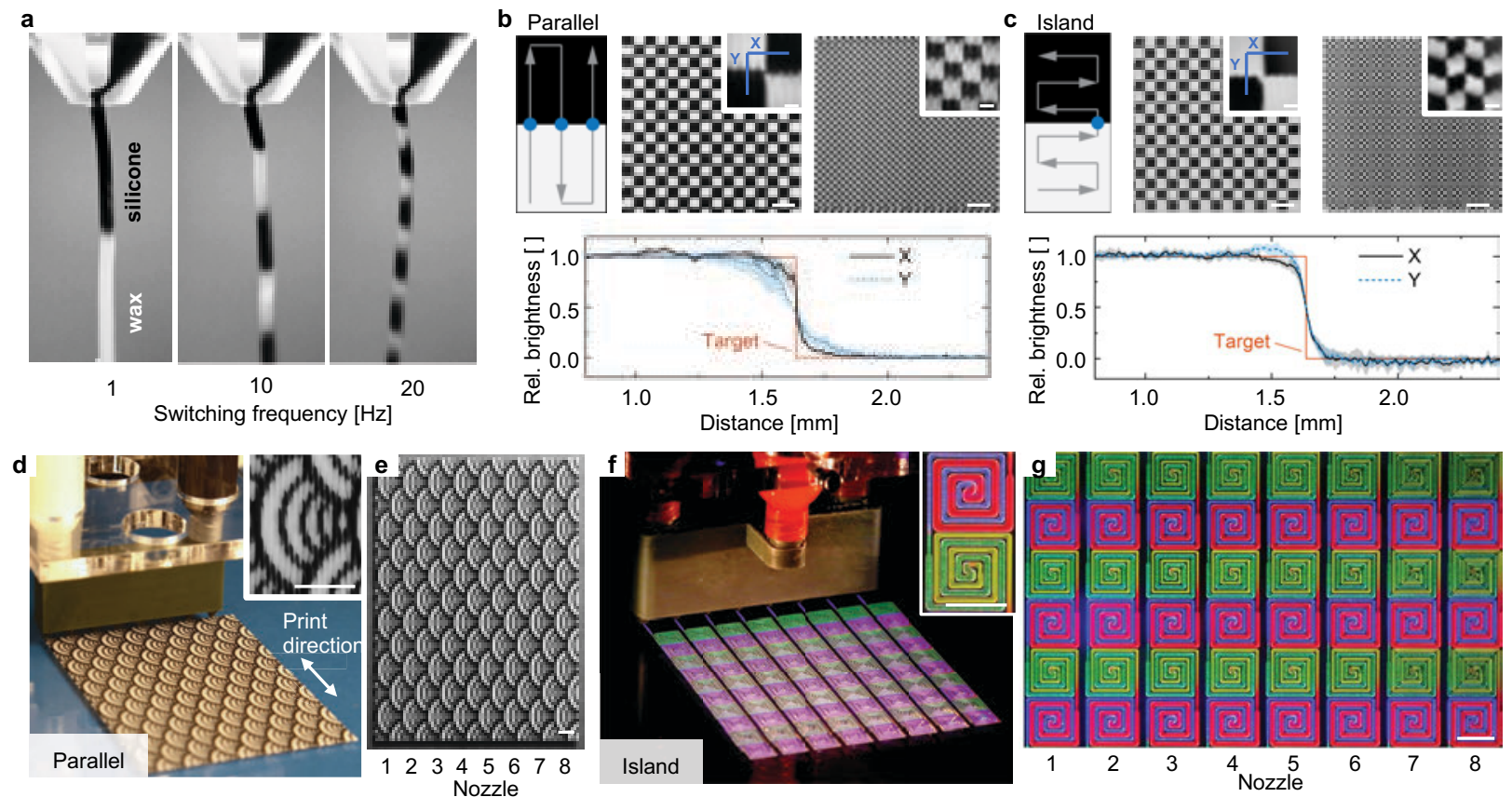

Figure 3 | Printing voxelated matter. a, A two-material nozzle producing a single continuous voxelated filament at increasing switching frequencies. b, c, Checkerboard patterns of silicone (black) and wax (white) manufactured by an $8 \times 1 \mathrm{MM} 3 \mathrm{D}$ printhead and the corresponding material transitions for parallel (b) and island (c) methods of printing. Shaded regions represent the standard deviation for $\mathrm{n}=6$ measurements. Scale bars in $\mathbf{b}, \mathbf{c}, 8 \mathrm{~mm}$, insets, $1 \mathrm{~mm}$. d, e, Parallel printing of an arbitrary pattern in silicone (black) and wax (white) using an asymmetric $8 \times 1$ two-material MM3D printhead. f, g, An $8 \times 1$ four-material MM3D printhead producing silicone spiral patterns by following a non-linear island print path. Scale bars in d-g $=5 \mathrm{~mm}$. 

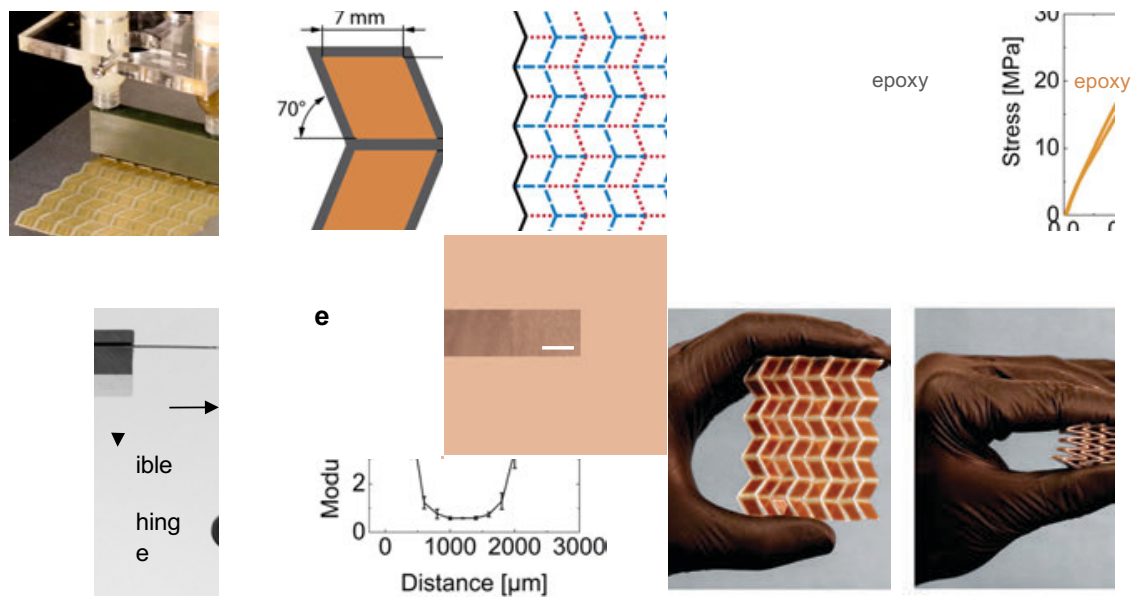

Figure 4 | MM3D printing of origami structures. a, I

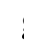

lattern using an $8 \times 1$ two-material MM3D printhead. b, Design of a Miura folding pattern. c, Stress-strain curves from $n=5$ tensile tests for flexible (grey, $0.61 \pm 0.16 \mathrm{MPa}$ ) and stiff (orange, $3.92 \pm 0.40 \mathrm{GPa}$ ) epoxies. d, Printed cantilevers (stiff, orange) and a hinge (flexible, grey) demonstrate patterned bending. e, Nanoindentation test results of an interfacial region measures a transition length of $\sim 400 \mu \mathrm{m}(10 \%$ to $90 \%)$. Error bars represent the standard deviation from $\mathrm{n}=6$ measurements. f, Folding behavior of an $8 \times 8$ Miura pattern. Print time $=2 \min 30 \mathrm{~s}$. 

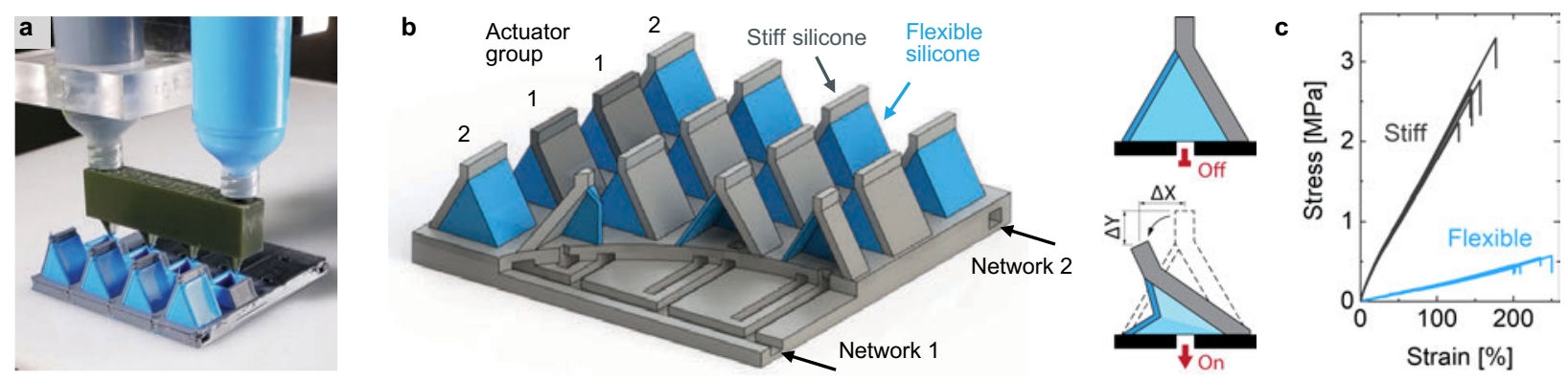

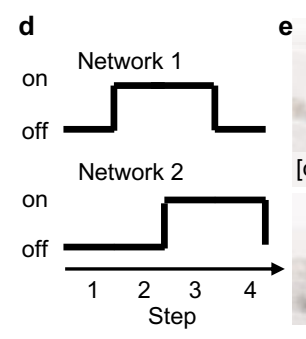

f

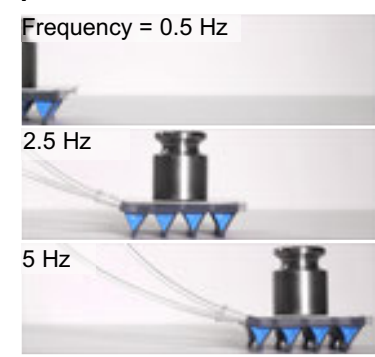

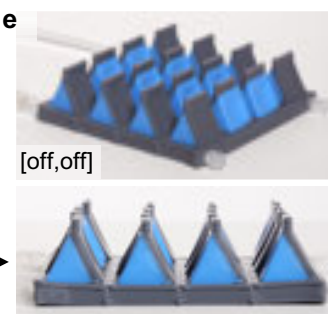

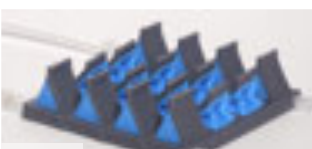

[on,off]
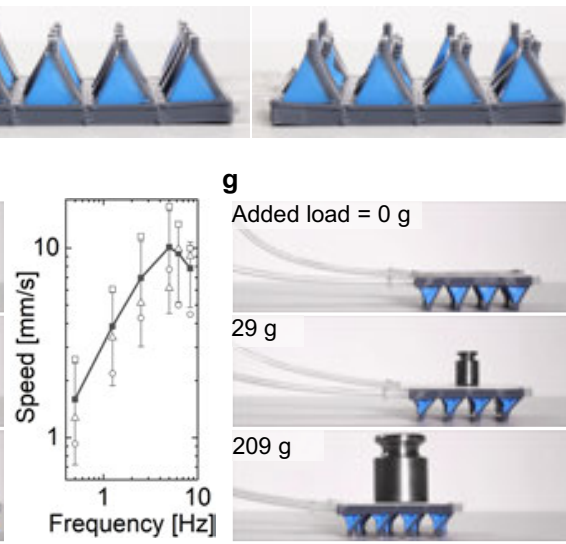

g

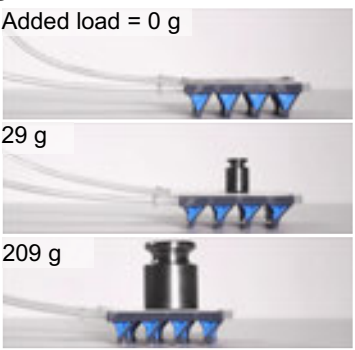

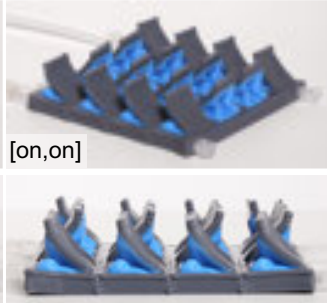

h

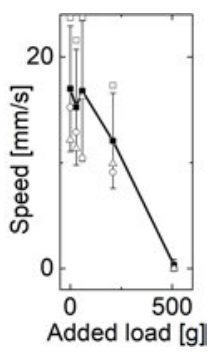

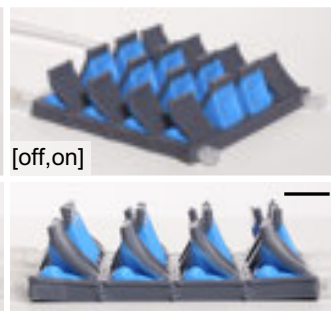

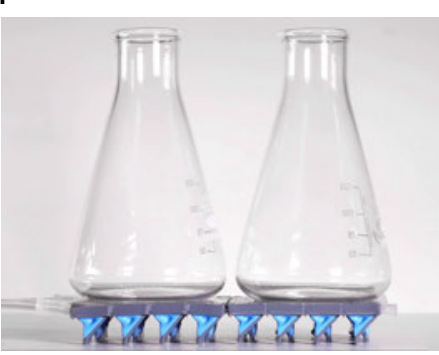

Figure 5 | MM3D printing of soft robotic walkers. a, MM3D printing of a soft-robotic millipede walker using a $4 \times 1$ two-material MM3D printhead. Print time $=17 \mathrm{~min}$. b, An illustration depicting the walker design elements. c, Stress-strain curves from $n=5$ tensile tests for stiff (grey, $1.81 \pm 0.05 \mathrm{MPa}$ ) and flexible (blue, $0.225 \pm 0.005 \mathrm{MPa}$ ) silicones. d, Actuation pattern for the two channel networks used to produce locomotion. Four actuation steps comprise one cycle. e, Photographs of the four actuation states for the steps described in d, depicting the buckling of the flexible silicone 'muscles' and the displacement of the stiff silicone 'legs.' Scale bar $=10 \mathrm{~mm}$. $\mathbf{f}$, Walking speed of the soft-robotic millipede as a function of cycle frequency. Added weight $=209 \mathrm{~g}$. g, Walking speed of the soft-robotic millipede as a function of added weight. Cycle frequency $=5 \mathrm{~Hz}$. Error bars for $\mathbf{f}, \mathbf{g}$, represent the standard deviation of speed measured for $\mathrm{n}=3$ independent walkers. $\mathbf{h}$, A pair of soft-robotic millipede modules connected in series to generate a longer walker, capable of transporting large labware. 


\section{Methods}

Materials: Silicone inks with different shear yield stresses were prepared by centrifugal mixing $(2,000$ rpm, 2 min, Flak-Tek, Inc.) of varying ratios of SE1700:Sylgard 184 (Dow Corning, Midland, MI, USA). Their color was modified by adding either $2 \% \mathrm{wt} / \mathrm{wt}$ pigment (Silc-Pig, Smooth-On Inc.) or 0.2\% wt/wt fluorescent dye (Risk Reactor, Inc., Santa Ana, CA, USA). Wax inks were prepared by mixing microcrystalline wax (SP-19, Strahl \& Pitsch, Inc., West Babylon, NY, USA) with petroleum jelly (Unilever, PLC) at a 4:6 weight ratio. The wax and petroleum jelly were combined in a covered glass beaker and heated in a furnace to $100^{\circ} \mathrm{C}$ until melted $(\sim 2 \mathrm{~h})$ followed by stirring and filtering through a $2 \mu \mathrm{m}$ glass fiber syringe-filter (Millex AP 20, MilliporeSigma, Burlington, MA, USA). The wax ink was stored at room temperature for $<1 \mathrm{mo}$ in a covered container. Immediately prior to use, the wax was mixed with $2 \% \mathrm{wt} / \mathrm{wt}$ pigment (Silc-Pig) in a centrifugal mixer (2,000 rpm, $2 \mathrm{~min})$. The flexible epoxy ink was prepared by mixing batches of $30 \mathrm{~g}$ of Epon 8130 (Momentive Performance Materials Inc., Waterford, NY, USA) with $4.5 \mathrm{~g}$ of CAB-O-SIL TS-530 fumed silica (Cabot Corporation, Boston, MA, USA), which was added in stepwise increments of $1.5 \mathrm{~g}$ to "max 100" containers (FlakTek Ink, Landrum, SC, USA). The solution was mixed in a DAC 600 VAC speed mixer (FlackTek, Inc, Landrum, SC, USA) in vacuum (20 Torr) after each step (90 s at $800 \mathrm{rpm}, 90 \mathrm{~s}$ at $1600 \mathrm{rpm}$, and $120 \mathrm{~s}$ at $2000 \mathrm{rpm}$ ). After allowing the inks to cool to room temperature, $3 \mathrm{~g}$ of Epikure 3272 curing agent (Hexion Inc., Columbus, OH, USA) was added and mixed for $30 \mathrm{~s}$ at $800 \mathrm{rpm}, 30 \mathrm{~s}$ at $1600 \mathrm{rpm}$, and $60 \mathrm{~s}$ at $2000 \mathrm{rpm}$. Similarly, the stiff epoxy ink was prepared by mixing $30 \mathrm{~g}$ of Epon 826 (Momentive Performance Materials Inc., Waterford, NY, USA) with $9 \mathrm{~g}$ nanoclay platelets (Nanocar 1.34 TN, Southern Clay Products, Inc., Gonzales, TX, USA) in stepwise increments of $3 \mathrm{~g}$ and $2.1 \mathrm{~g}$ dimethyl methyl phosphonate (Sigma Aldrich, St. Louis, MO, USA). $1.5 \mathrm{~g}$ of Basionics VS03 curing agent (BASF, Ludwigshafen, Germany) was added after the ink reaches room temperature and mixed in the speed mixer accordingly. The same mixing cycles as used for the flexible ink were used. For the soft robots, the stiff silicone ink was prepared by combining SE1700 base and catalyst at a 10:1 weight ratio, then adding 2\% wt/wt pigment (Silc-Pig) in a centrifugal mixer (2000 rpm, $2 \mathrm{~min})$. The flexible silicone ink was prepared by first mixing SE1700 base (40 g), catalyst (1.6 g), and pigments ( $1 \mathrm{~g})$ in a speed mixer (2000 rpm, 2 min), followed by adding Sylgard 527 Part A (3.4 g) and Part B (3.4 g) and mixing again (2000 rpm, $2 \mathrm{~min})$.

Gelatin inks, $15 \% \mathrm{wt} / \mathrm{vol}$, were prepared by stirring gelatin (type A; 300 bloom from porcine skin; Sigma) with phosphate buffered saline (PBS) without calcium or magnesium in a covered Erlenmeyer flask, at $85^{\circ} \mathrm{C}$ for $12 \mathrm{~h}$. The molten gelatin was then brought to $\mathrm{pH} 7.5$ by dropwise addition of $1 \mathrm{M} \mathrm{NaOH}$ and filtered using a $0.2 \mu \mathrm{m}$ vacuum filter. The molten gelatin was then diluted in PBS to $7.5 \% \mathrm{wt} / \mathrm{vol}$ and colored by addition of $1 \% \mathrm{vol} / \mathrm{vol}$ food coloring. The molten gelatin is directly pipetted into a syringe, 
covered, and allowed to undergo gelation by incubating at $4^{\circ} \mathrm{C}$. Kept sterile, the syringe could be stored for up to 6 months at $4^{\circ} \mathrm{C}$.

Multi-Material Multinozzle 3D Printheads. The MM3D printheads were designed using 3D computeraided design software (Dassault Systèmes SolidWorks Corporation, Waltham, MA, USA). The model was converted to a .stl file and printed using a stereolithography 3D printer (Perfactory Aureus, Envisiontec, Inc., Dearborn, MI, USA). For all high resolution MM3D printheads, HTM140_v2 green resin was used, with a layer height of $50 \mu \mathrm{M}$ and a calibrated power of $600 \mathrm{~mW}$. Alternatively, for visualization purposes, clear resin (EShell 300) was used with a layer height of $100 \mu \mathrm{m}$ and a calibrated power of $120 \mathrm{~mW}$. Once printed, the nozzles were rinsed with isopropyl alcohol using a syringe to flush the channels. The nozzles were then dried under a stream of air and stored in the dark until ready for use.

MM3D Printheads were attached to a set of ink-filled syringes that were mounted on a six-axis air bearing linear motion controller, described previously ${ }^{21}$. Pressure was supplied to the syringes via digital pressure regulators (Ultimus V, Nordson EFD, Inc., Westlake, OH, USA) that were gated through pneumatic solenoid valves (VQD1151, SMC Corp., Tokyo, Japan). The solenoid valves were operated by 0-24V digital relays (WAGO, Inc., Germantown, WI, USA) that were synchronized to the print motion through an NPAC A3200 motion controller (Aerotech Inc., Pittsburgh, PA, USA).

Rheological Measurements: Ink rheology was measured using a controlled stress rheometer (TA Instruments, New Castle, DE, USA) equipped with a cone-plate geometry composed of a diameter of 40mm and a cone angle of $2^{\circ}$. After inks were loaded into the cone-plate gap, excess ink was trimmed using a spatula. Apparent viscosities were determined at $20^{\circ} \mathrm{C}$ by carrying out shear-rate sweeps using shear rates of $\sim 1 \mathrm{~s}^{-1}$ to $\sim 100 \mathrm{~s}^{-1}$. The data were fitted using a Herschel-Bulkley model to determine the consistency, flow index, and shear yield stress values for each ink. The storage and loss moduli for epoxy inks were measured across a range of oscillation frequencies from $0.1 \mathrm{~Hz}$ to $10 \mathrm{~Hz}$, applying a shear stress of $10 \mathrm{~Pa}$. Next, oscillatory amplitude sweeps were measured using strains of $0.01 \%$ to $1,000 \%$, using a frequency of $1 \mathrm{~Hz}$. The experiments were conducted within 30 minutes of preparing the inks. To assess ink pot life, the complex viscosity was measured for 15 hours at $1 \%$ strain applied at a frequency of $2 \mathrm{~Hz}$.

To measure static yield stresses, silicone inks of various compositions were loaded into metal tubing with inner diameters of $0.84 \mathrm{~mm}$ and with lengths ranging from $12.35 \mathrm{~mm}$ to $44.10 \mathrm{~mm}$. A pressure controller (Ultimus V, Nordson EFD Inc.) was connected in series, via water-filled tubing, with a microfluidic flowmeter (GeSiM GmbH, Großerkmannsdorf, Germany). The applied pressure was increased stepwise, and yielding was determined to occur at the onset of flow $\left(Q_{s}>0.1 \mu 1 / \mathrm{min}\right)$. This yield pressure was measured for each tube length, and the gradient of the line was used to calculate the static shear yield stress 
of the ink. To measure $Q_{f}^{\max }$ for different combinations of static and flow materials, a gas-tight glass syringe (Hamilton Inc., Reno, NV, USA) was loaded with a silicone ink via centrifugation and was mounted onto a syringe pump. Care was taken to avoid introducing air bubbles upon insertion of the plunger. The 'flow' side of a model MM3D nozzle was connected to the syringe, while the 'static' side of the printhead, loaded with a second silicone ink, was connected via water-filled tubing to the microfluidic flowmeter. The flow rate was ramped step-wise until an onset of flow $\left(Q_{s}>0.1 \mu \mathrm{l} / \mathrm{min}\right)$ was detected by the microfluidic flowmeter, at which point, the static material was deemed to have yielded.

Printing Voxelated Matter: Both Muira origami patterns and 3D voxelated architectures were fabricated on an Aerotech stage (Aerotech, Inc., Pittsburgh, PA, USA) fitted with EFD Ultimus V pressure regulators (Nordson, Inc., Westlake, OH, USA). Five or more dogbone and hinge specimens are printed onto glass slides with a single bimaterial switching nozzle at a print speed of $8 \mathrm{~mm} / \mathrm{s}$ and pressures of $51 \mathrm{psi}$ and 59 psi for the flexible and stiff inks, respectively. The Miura patterns are printed with a print speed of $10 \mathrm{~mm} / \mathrm{s}$ and pressures of $55 \mathrm{psi}$ and $66 \mathrm{psi}$, respectively. The soft robots were printed with a print speed of $40 \mathrm{~mm} / \mathrm{s}$ and the flexible and stiff silicones were printed at pressures of 53 psi and 62 psi, respectively. The samples are cured in a furnace for $18 \mathrm{~h}$ at a constant temperature of $100^{\circ} \mathrm{C}$ with no ramping up or down. The dogbone specimen tests were conducted according to ASTM D638-10 on an Instron 5566 Universal Testing Machine fitted with pneumatic grippers mounted on $100 \mathrm{~N}$ and $1000 \mathrm{~N}$ static load cells (depending on the maximum required load) at a testing speed of $10 \mathrm{~mm} \mathrm{~s}^{-1}$ (Instron, High Wycombe, UK). On the same machine, the cyclic tests were conducted at a frequency of $6 \mathrm{~Hz}$ with an amplitude resulting in an angular displacement ranging from $18^{\circ}$ to $142^{\circ}$ (Extended Data Fig. 9e,f). The soft robots were actuated using a vacuum pressure that was gated via a pair pneumatic solenoid valves (VQD1151, SMC Corp., Tokyo, Japan). The solenoid valves were controlled as described for operating the MM3D printheads. To measure the walking speed, videos were analyzed for three different soft-robotic millipedes to estimate the time taken for each to move by one body-length. Printer motion was controlled via GCode that was either coded manually or, for more complex print patterns, generated automatically from bitmap files using a custom script (MATLAB, Mathworks, Inc., Natick, MA). Bitmaps were designed manually or could be generated from a stereolithography (.stl) file using Freesteel Slicer. The custom MATLAB script read the bitmap file and generated a bidirectional parallel rastering print path, while simultaneously switching material on-thefly to produce the pattern. The exact switching location was controlled by an offset variable which was tuned by bidirectionally printing vertical lines to visualize alignment. 2D MM3D Printheads were physically aligned to the print substrates (glass slides) using a two-axis tilt stage. 
Scaling Laws for MM3D Printing: Consider an MM3D printhead that is extruding filaments with diameter $D$, is translating at a print speed $v$, and is switching materials at a switching frequency $f$ (Extended Data Fig. 1e,f). The resulting voxels will be roughly cylindrical, with diameter $D$, and an axial length given by:

$$
l_{v}=\frac{v}{f}
$$

The nozzle has a maximum switching frequency $f_{\max }$, which is limited by the cycling time of the pneumatic solenoids and the resistance and capacitance of the air channels and syringe. If the nozzle operates at its maximum switching frequency and the print speed is set such that $v=D f_{\max }$, then the cylindrical voxels become roughly cubic, i.e. $l_{v}=D$. The characteristic build time $(t)$ required to print an arbitrary voxelated part of characteristic length $L$, using an array of $N$ nozzles is given by:

$$
t \sim \frac{L^{3}}{D^{2} v N}=\frac{L^{3}}{D^{3} f_{\max } N}
$$

Here, the cubic dependency on part size can be ameliorated by using a printhead that is scaled with part size, i.e.:

$$
N=\left(\frac{L}{l}\right)^{k}
$$

where $l$ is the spacing between nozzles and $k \in\{0,1,2\}$ represents the dimensionality of the nozzle array. Given these parameters, we can vastly reduce the total build time from $O\left(L^{3}\right)$ for a single-nozzle, to $O\left(L^{2}\right)$ for a 1D nozzle array, or $O(L)$ for a 2D nozzle array (Fig. $1 \mathrm{~A})$ :

$$
t \sim \frac{L^{3-k} l^{k}}{D^{2} v}
$$

By decreasing the distance between individual nozzles within the printheads, i.e. the nozzle spacing parameter $l$, we can reduce the build time required for a given 3D object. However, if each nozzle is restricted to printing the same material at any given time, the part complexity is decreased due to periodicity constraints; i.e., as the spacing is decreased, less information can be encoded within the printed object (Extended Data Fig. 3). Specifically, the number of bits of information that can be encoded into a cube of side length $L$, by a nozzle printing at speed $v$ that can switch between 1 -of- $S_{\text {ink }}$ materials at a maximum frequency $f_{\max }$ is given by:

$$
I=\frac{L^{3-k} l^{k}}{D^{2} v} f_{\text {max }} \log _{2} S_{\text {ink }}=t f_{\max } \log _{2} S_{\text {ink }}
$$


Importantly, this analysis suggests that implementation of MM3D printheads with high $S_{i n k}, N$, and $f_{\max }$ would enable the rapid construction of complex 3D architectures with a minimum voxel size approaching $D^{3}$.

Deriving an analytical model for viscoelastic inks at a junction: Consider the force balance for a static plug of complex fluid in a cylindrical channel. The critical pressure, $p_{c r}$, at which the plug will yield is given by:

$$
p_{c r}=\frac{4 \tau_{y s} L_{C s}}{d}
$$

where $\tau_{y s} \in\left\{\tau_{y l}, \tau_{y 2}\right\}$ is the shear yield stress of the static material, and $L_{C s} \in\left\{L_{C l}, L_{C 2}\right\}$ and $d$ are the length and diameter of the static channel respectively ${ }^{30}$. Note that upon toggling the actuation states of the pneumatic solenoid valves, the material in the static channel begins to flow under pressure, whereas the previously flowing material now becomes static. In addition, a compensatory forward pressure $\left(p_{s}>p_{a t m}\right)$ could be applied to the inlet of the static side to resist yielding at a higher $p_{j}$, in which case backflow is prevented so long as:

$$
\left(p_{j}-p_{s}\right)<p_{c r}
$$

The junction back pressure, $p_{j}$, results from the flow of the flow material, $Q_{f}$. For a HerschelBulkley material flowing through a cylindrical pipe, Chilton and Stainsby provide the following closedform solution ${ }^{30}$ :

$$
p_{j}=\frac{4 k_{f} L_{o}}{d}\left(\frac{8 V_{f}}{d}\right)^{n_{f}}\left(\frac{3 n_{f}+1}{4 n_{f}}\right)^{n_{f}}\left(\frac{1}{1-X}\right)\left(\frac{1}{1-a X-b X^{2}-c X^{3}}\right)^{n_{f}}
$$

where:

$$
a=\frac{1}{2 n_{f}+1} ; b=\frac{2 n_{f}}{\left(n_{f}+1\right)\left(2 n_{f}+1\right)} ; c=\frac{2 n_{f}{ }^{2}}{\left(n_{f}+1\right)\left(2 n_{f}+1\right)} ; X=\frac{4 L_{o} \tau_{y f}}{d p_{j}}
$$

and $k_{f}, n_{f}$, and $\tau_{y, f}$ are the Herschel-Bulkley consistency index, flow index, and shear-yield stress, respectively, for the flowing material, and $L_{0}$ is the outlet channel length. To solve for $p_{j}$ for a given mean velocity $V_{f}$, this implicit equation requires an iterative solution. However, for a given $p_{j}$, we can rearrange this equation to provide an explicit solution for $V_{f}$.

$$
V_{f}=\frac{d}{8}\left(\frac{d p_{j}}{4 k_{f} L_{o}}\left(\frac{4 n_{f}}{3 n_{f}+1}\right)^{n_{f}}(1-X)\left(1-a X-b X^{2}-c X^{3}\right)^{n_{f}}\right)^{\frac{1}{n_{f}}}
$$


At the critical mean velocity, $V_{c r}$, the junction pressure is sufficient to cause yielding of the static material: $p_{j}=p_{c r}\left(\right.$ assuming $\left.p_{s}=p_{a t m}\right)$. By substituting $p_{j}$ in Eq. 12 and Eq. 13 for $p_{c r}$ in Eq. 6, we derive an explicit solution for $V_{c r}$ :

$$
V_{c r}=\frac{d}{8}\left(\frac{\tau_{y s} L_{C s}}{k_{f} L_{o}}\left(\frac{4 n_{f}}{3 n_{f}+1}\right)^{n_{f}}\left(1-X_{c r}\right)\left(1-a X_{c r}-b X_{c r}^{2}-c X_{c r}^{3}\right)^{n_{f}}\right)^{\frac{1}{n_{f}}}
$$

where:

$$
X_{c r}=\frac{4 L_{o} \tau_{y f}}{d p_{c r}}=\frac{4 d L_{o} \tau_{y f}}{4 d L_{s} \tau_{y s}}=\frac{L_{o} \tau_{y f}}{L_{C s} \tau_{y s}}
$$

This critical print speed of the flow material can be related to the maximum volumetric extrusion rate by:

$$
Q_{f}^{\max }=\frac{\pi d^{2}}{4} V_{c r}
$$

Eq. 14 predicts a zero at $X_{c r}=1$, whereupon the static material yields with any non-zero flow rate of the flow material. These zeroes are shown as dotted lines for various selections of flow materials on Fig. $2 \mathrm{f}$. The constraint that $X_{c r}<1$ results in a necessary geometric and material design criterion that must be obeyed for all combinations of flow and static materials for the successful operation of an MM3D printhead (assuming a constant channel diameter):

$$
\frac{\tau_{y f}}{\tau_{y s}}<\frac{L_{C s}}{L_{o}}
$$

Importantly, Eq. 17 shows that non-yield stress materials, i.e., $\tau_{y s}=0$, cannot be used in a pressure-driven MM3D system.

In general, the maximum constant print speed, $v_{c r}$, for MM3D printing of a set of materials, $M$, is given by the minimum $v_{c r}$ for all combinations of flow and static materials:

$$
v_{c r}=\min _{f \in M, s \in M} V_{c r}(f, s)
$$

Given that such materials may exhibit a wide range of flow behavior, this could lead to a 'weakest-link' material that limits print speed. While $v_{c r}$ can be made arbitrarily large by making $L_{C 1} \ldots L_{C S_{\text {ink }}} \rightarrow \infty$ (assuming non-zero shear yield stresses), this would result in prohibitively high resistances to flow since viscoelastic DIW inks typically require high printing pressures (often $>>100 \mathrm{psi}$ ). 


\section{Additional References}

30. Chilton, R. A. \& Stainsby, R. Pressure loss equations for laminar and turbulent non-Newtonian pipe flow. J. Hydraul. Eng. 124, 522-529 (1998). 


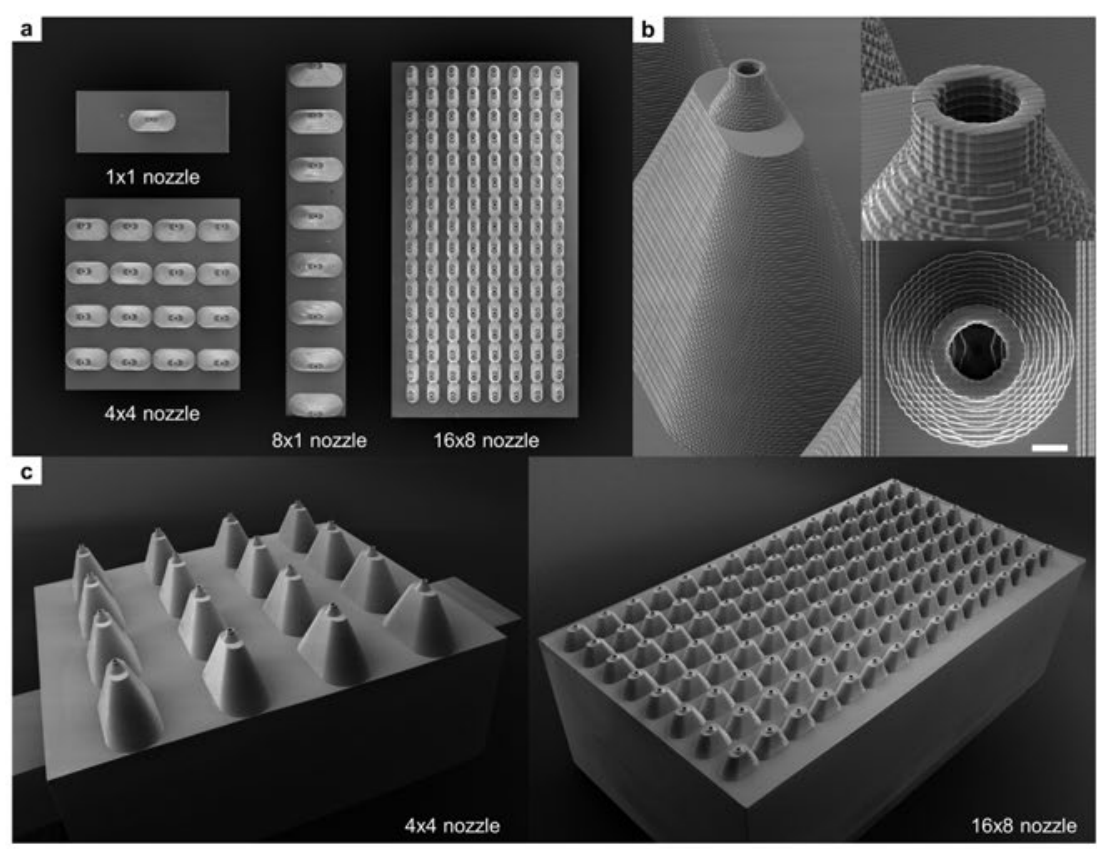

d
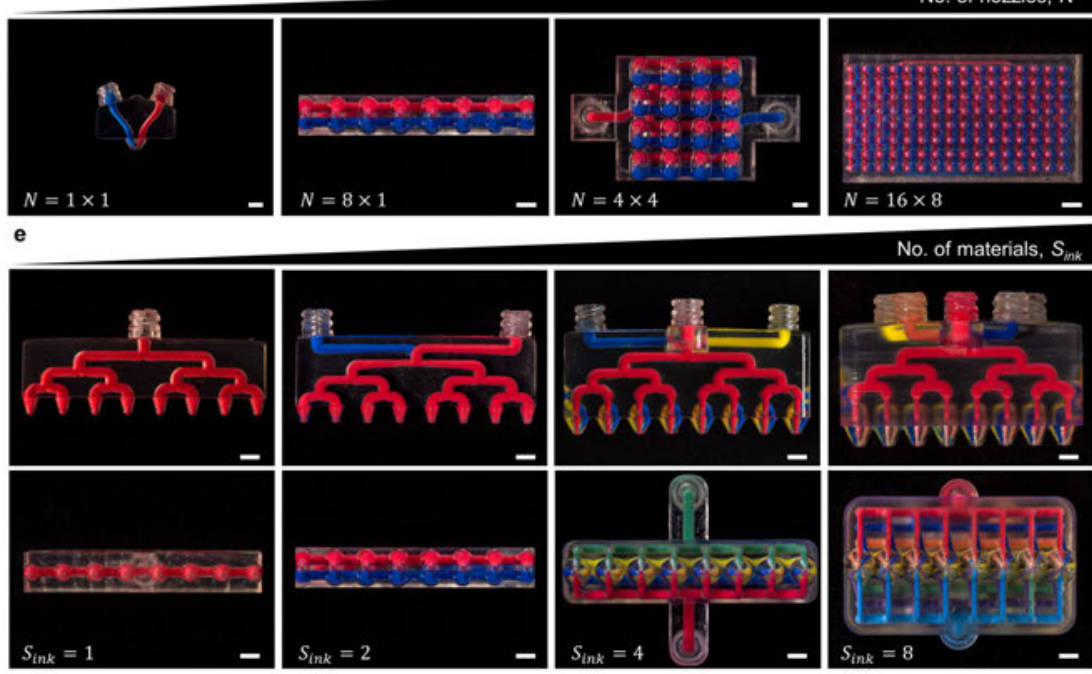

Extended Data Figure 1 | SEM images and photographs of MM3D printheads with varying number of nozzles and materials. a, SEM images (top down view) of $0 \mathrm{D}(1 \times 1), 1 \mathrm{D}(8 \times 1)$, and $2 \mathrm{D}(4 \times 4 ; 16 \times 8)$ nozzle arrays for two-material MM3D printing. b, Higher magnification SEM images of these printheads showing an individual nozzle outlet (tilted and top down views). Scale bars $=200 \mu \mathrm{m}$. c, SEM images (tilted views) of $4 \times 4$ and $16 \times 8$ two-material MM3D printheads. d, Photographs of MM3D printheads showing $N=1 \times 1,8 \times 1,4 \times 4$, and $16 \times 8$ designs, which increase from 1 to 128 nozzles per printhead (from left to right), where $s_{\text {ink }}=2$. e, Photographs showing side (top row) and top (bottom row) views of MM3D printheads with increasing materials being delivered to each nozzle, where $N=8 \times 1$. Scale bars $=5 \mathrm{~mm}$. 

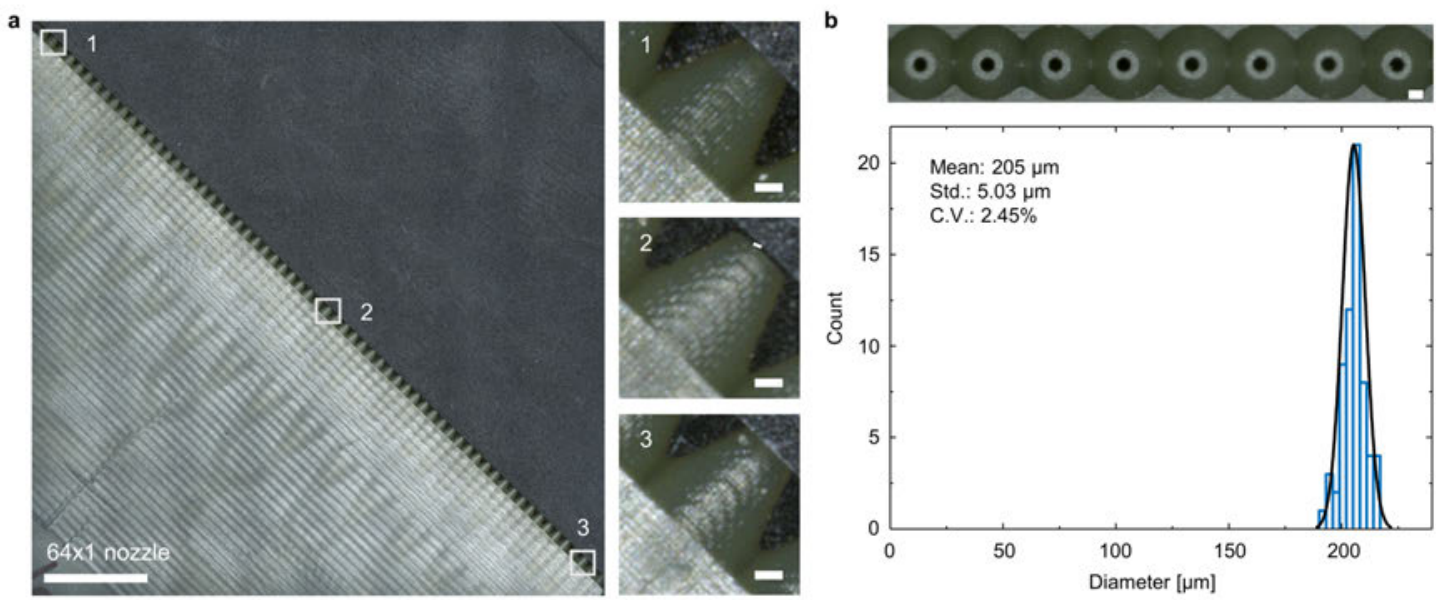

Extended Data Figure 2 | Printhead flatness and hole diameter repeatability. a, A $64 \times 1$ MM3D printhead, fabricated using stereolithography. The printhead has a flatness of $8 \mu \mathrm{m}$ across the total length of the nozzle $(60 \mathrm{~mm})$. Scale bars left $=8 \mathrm{~mm}$; right $=200 \mu \mathrm{m}$. b, A histogram showing diameter of nozzle outlets. The actual nozzle outlets (diameter $=205 \mu \mathrm{m} \pm 5.03 \mu \mathrm{m}$ ) are slightly larger than their designed diameter of $200 \mu \mathrm{m}$. (Note: The top image represents a representative view of 8 nozzle outlets out of a total of 64 nozzle outlets in this 1D nozzle array). Scale bar $=200 \mu \mathrm{m}$. 

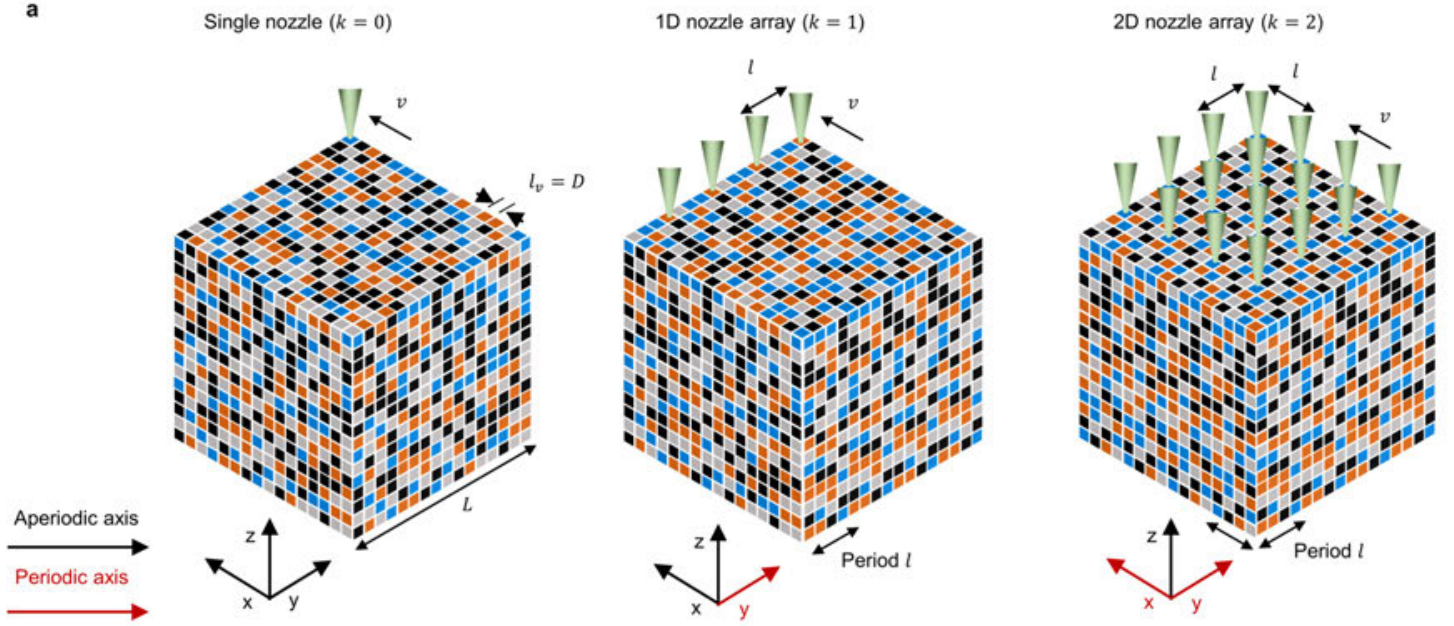

Print Time $\quad t=\frac{L^{3}}{D^{2} v}$

$t=\frac{L^{2} l}{D^{2} v}$

$$
t=\frac{L l^{2}}{D^{2} v}
$$

b

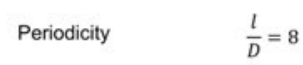

$\frac{l}{D}=4$
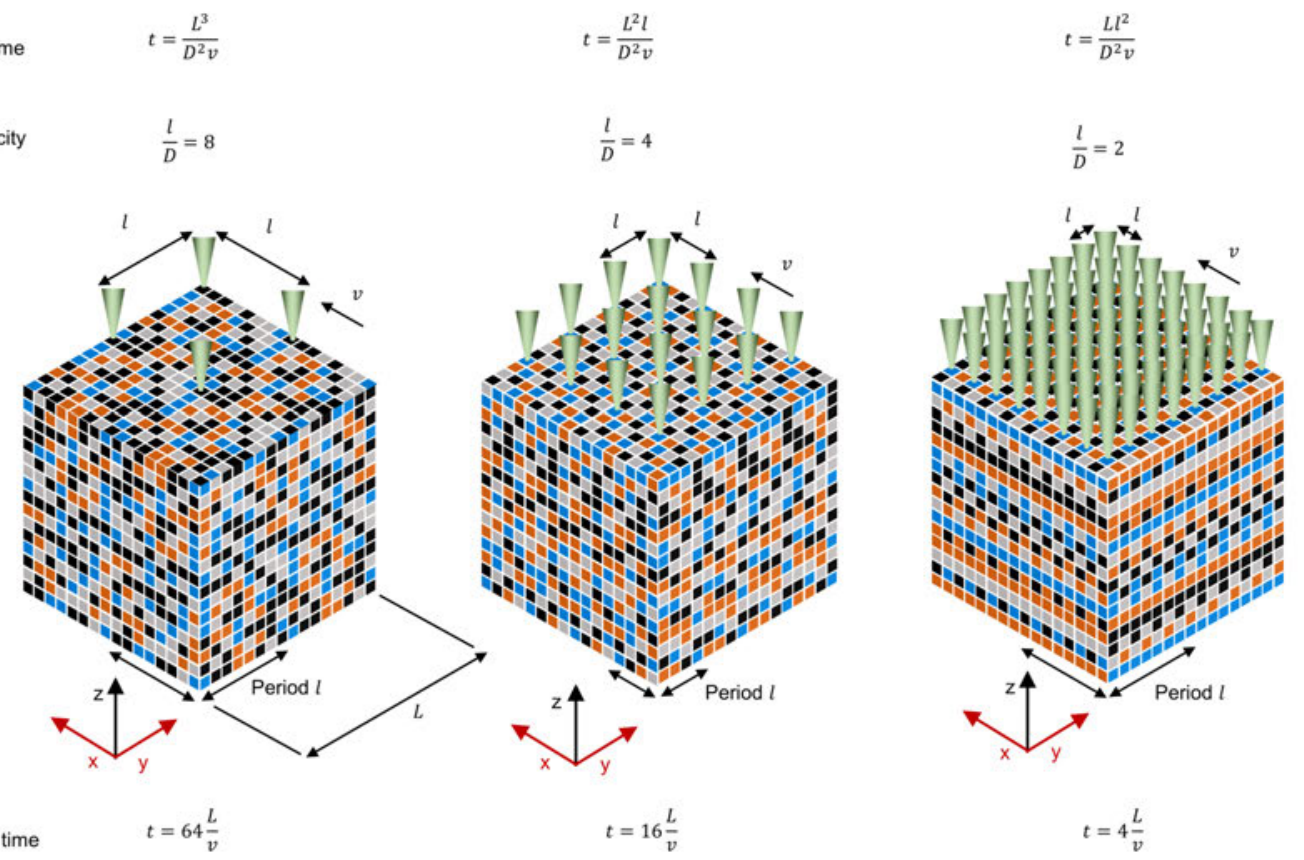

Print time

$$
t=64 \frac{L}{v}
$$$$
t=16 \frac{L}{v}
$$

Extended Data Figure 3 | Scaling laws for MM3D printing. a, The periodicity and print time depend on the dimensionality, $k$, of the MM3D printhead. A single (0D) nozzle (left) has no periodic axis, providing the greatest complexity, but longest print time. A $1 \mathrm{D}$ nozzle array is constrained to be periodic in one axis, but reduces the print time from $O\left(L^{3}\right)$ to $O\left(L^{2}\right)$. The 2D nozzle array is periodic in two axes, but results in the shortest print time of $O(L)$. b. The complexity and print time also depend on the spacing between nozzles in each printhead design. $L=$ part scale, $D=$ nozzle diameter, $l_{v}=$ voxel length, $v=$ print speed, $t=$ print time, $l=$ nozzle spacing. 

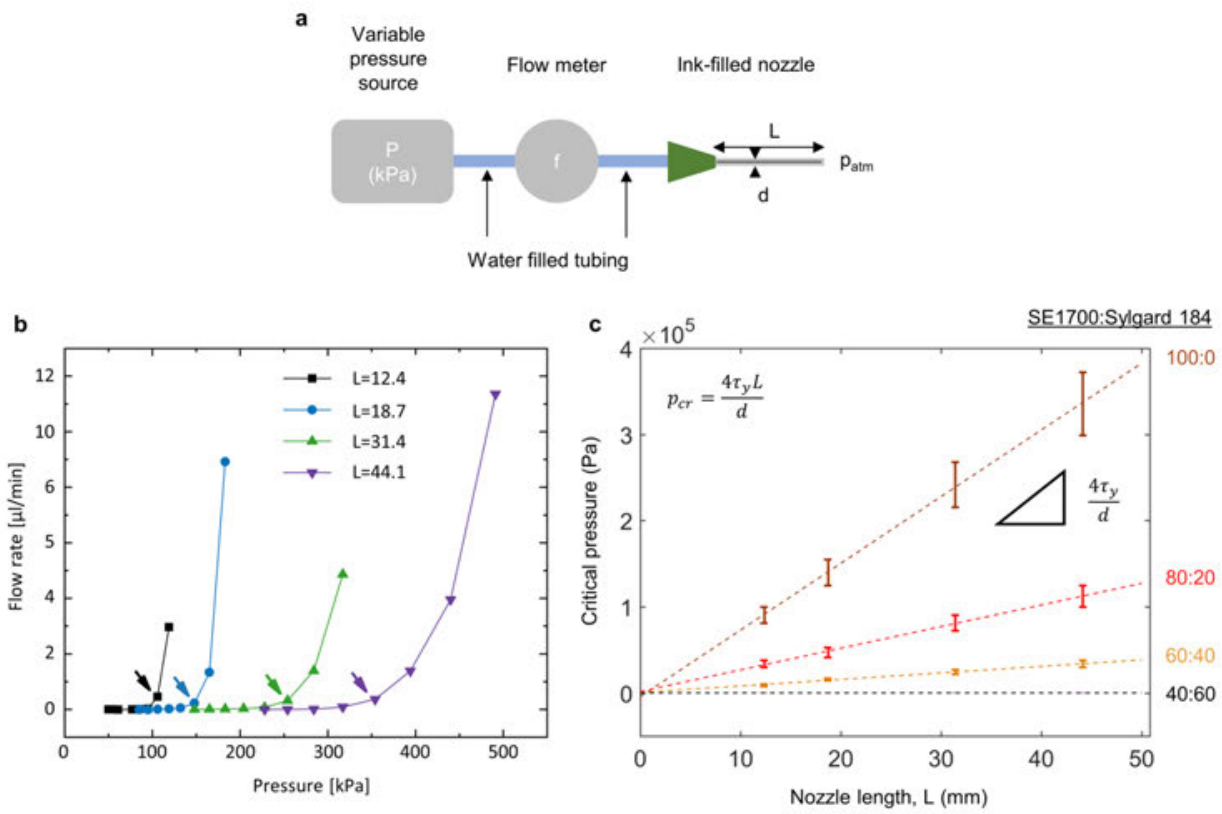

Extended Data Figure 4 | Measurements of critical pressure, $p_{c r}$, for various nozzle lengths and materials. a, A variable pressure source is placed in series with a microfluidic flow meter and an ink-filled nozzle to measure the critical pressure for a given nozzle tip length, L. b, A representative set of measurements of flow rate versus pressure. The arrows represent the critical pressures for each material, at which yielding was first observed. c, The measured critical pressures analyzed for different silicone elastomer inks (varying SE1700:Sylgard 184 ratios) as a function of the nozzle length, where the dashed lines are fitted by linear regression and error bars represent the resolution of pressure steps used for analysis $(\mathrm{n}=1)$.
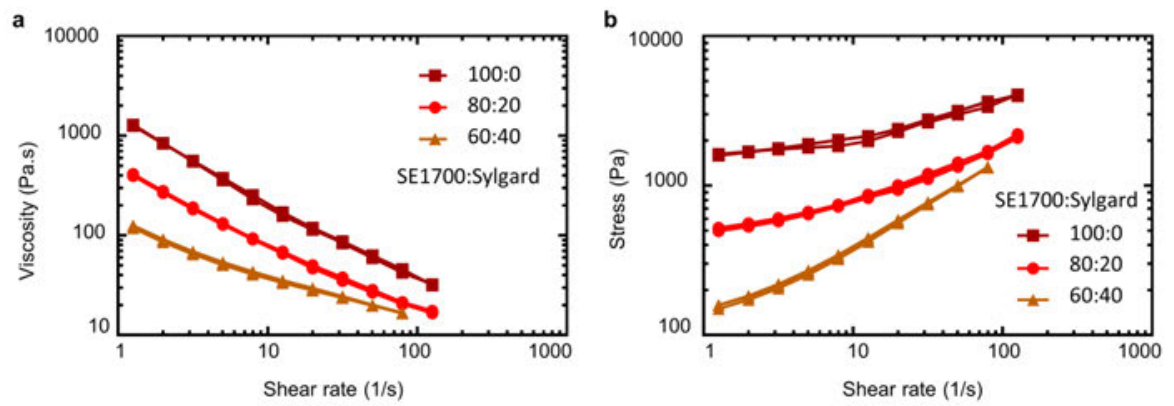

Extended Data Figure 5 | Rheological properties of different silicone inks. Log-log plots of a, apparent viscosity and $\mathbf{b}$, shear stress as a function of shear rate for silicone elastomer inks with different compositions (i.e., SE1700:S184 Sylgard ratios), fit to the Herschel-Bulkley model. Data represents $n=3$ independent experiments per ink. 
a

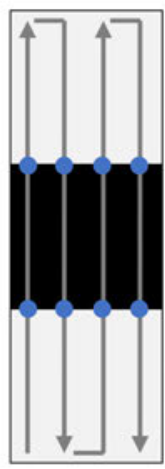

$\square$ Material A

Material B

- Material switching
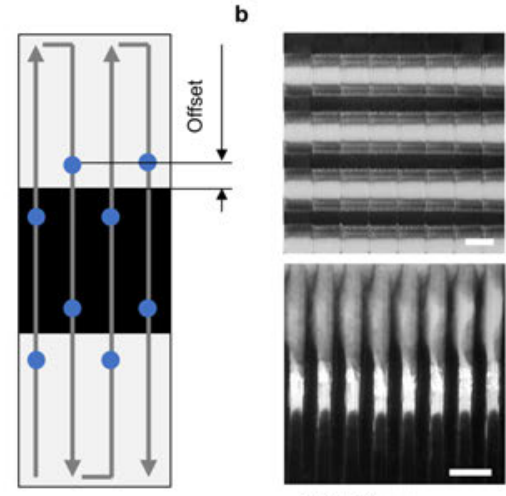

Offset: $0 \mathrm{~mm}$

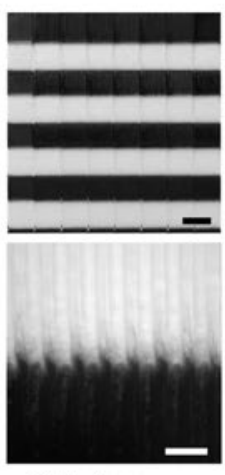

Offset: $6 \mathrm{~mm}$

c
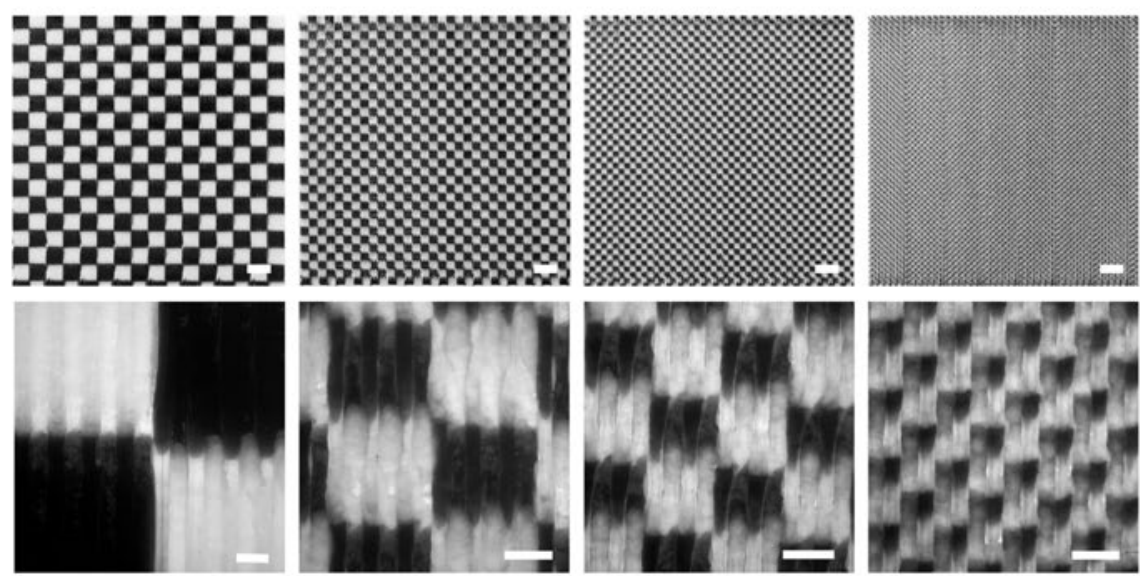

Extended Data Figure 6 | Offset length and print path design. a, b, Due to a finite output channel length, $L_{o}$, and inertance in the system, a delay occurs when switching between materials that can be corrected by introducing an offset length, as shown schematically (a) and during parallel printing of horizontal lines in silicone (black) and wax (white) (b). c, Offset-corrected printing of resolution grids using silicone and wax inks showing increasingly smaller features (from left to right), and thus high switching frequencies. Scale bars $(\mathrm{b}, \mathrm{c})$ : bottom $=1 \mathrm{~mm}$, top $=5 \mathrm{~mm}$. 


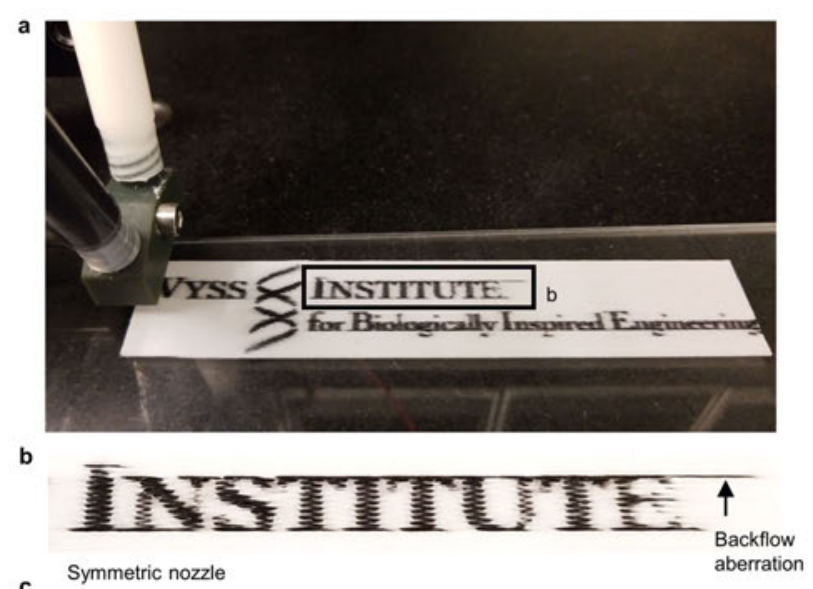

c

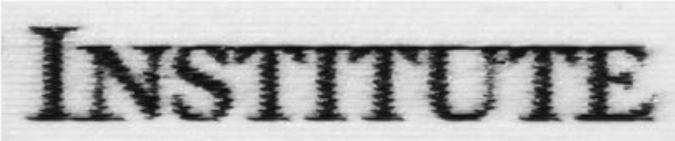

Asymmetric nozzle

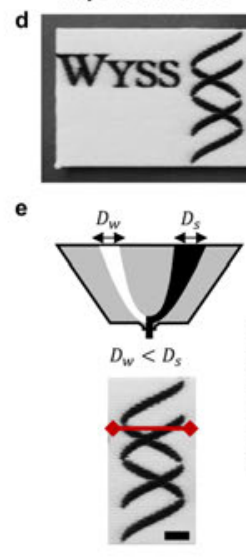

\section{INSTITUTE}
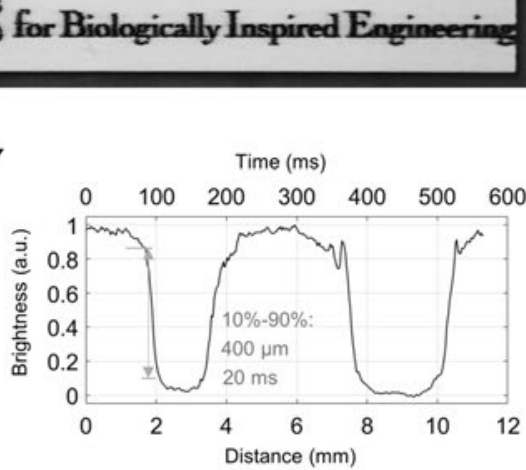

Extended Data Figure 7 | Backflow aberration correction using an asymmetric nozzle. a, b Printing a two-material pattern with a symmetric $0 \mathrm{D}$ nozzle that switches between two materials (a) induces backflow aberrations (b), due to printhead operation at $v>V_{c r}$ for the silicone (black). The aberration occurs due to backflow of the stiffer black silicone into the channel with the softer white wax. Hence, after a long period of extruding black ink, undesired black ink flows out upon switching to the wax channel. (c-e) An asymmetric nozzle, in which the wax channel is narrower than the silicone channel, prevents the occurrence of backflow aberrations. 

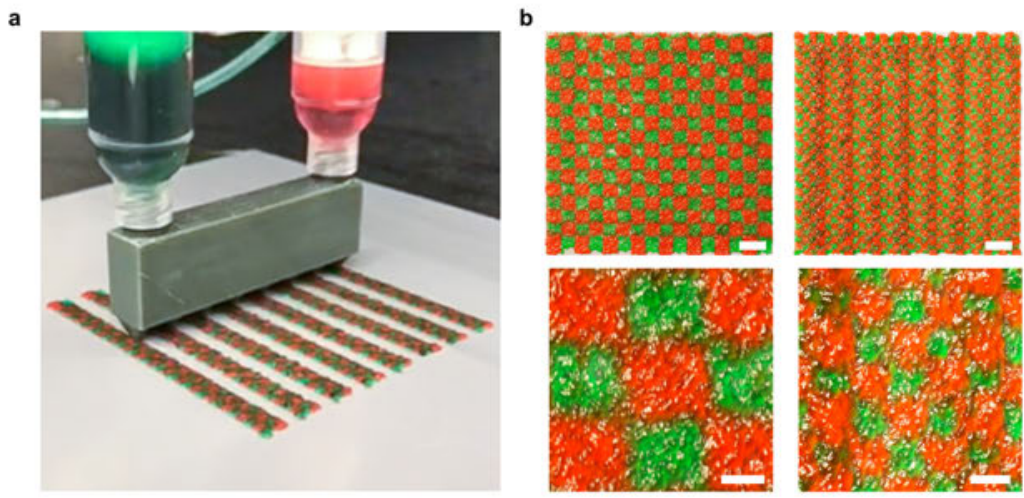

Extended Data Figure 8 | MM3D printing of gelatin hydrogels. a, Photograph of an MM3D printhead $\left(N=8 \times 1, S_{i n k}=2\right)$ printing two $7.5 \%$ gelatin inks dyed with red and green food coloring, respectively. $\mathbf{b}$, A parallel print path strategy is used to manufacture gelatin checkerboards with square sizes of $4 \mathrm{~mm} \times 4$ $\mathrm{mm}$ (left) and $2 \mathrm{~mm} \times 2 \mathrm{~mm}$ (right). Scale bars: top row $=8 \mathrm{~mm}$; bottom row $=2 \mathrm{~mm}$. 
a

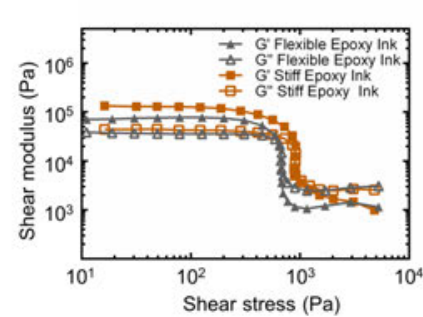

c
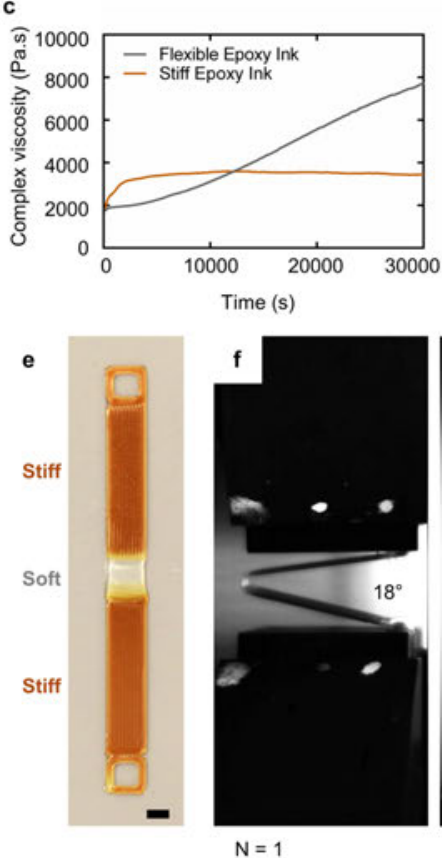

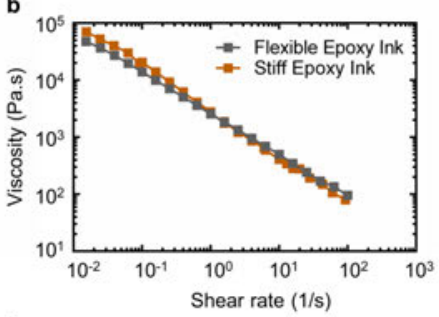

$d$
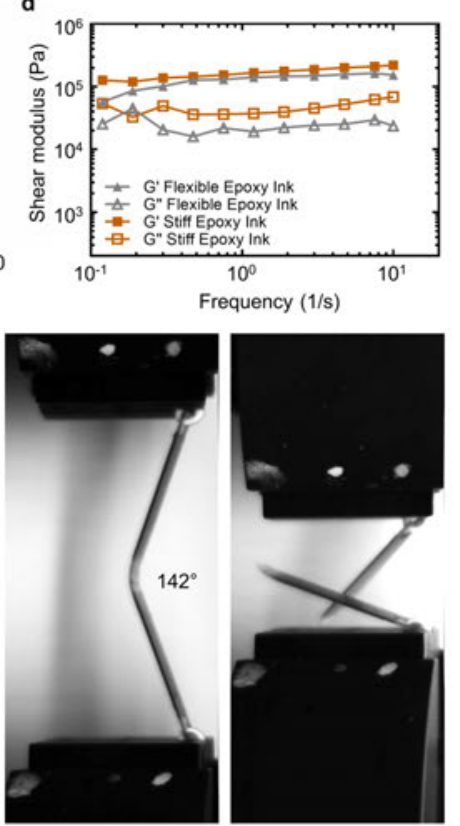

$N=1$

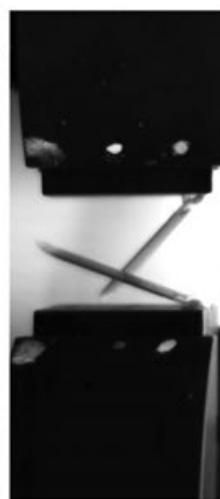

$N=1632$

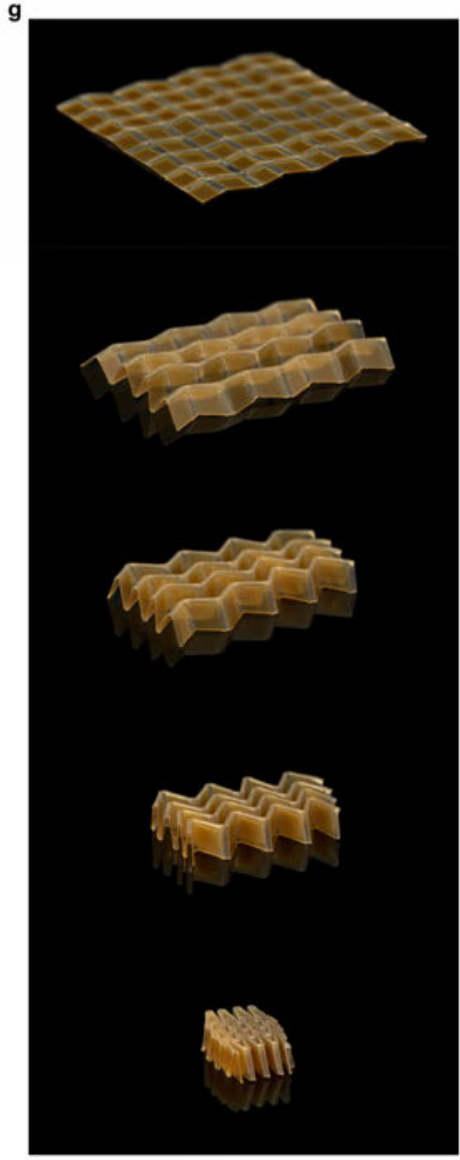

Extended Data Figure 9 | Rheology, mechanical testing, and MM3D printing of flexible and stiff epoxy inks. a, Storage $\left(G^{\prime}\right)$ and loss $\left(G^{\prime \prime}\right)$ moduli versus shear stress for flexible (grey) and stiff (orange) epoxy inks. b, Apparent viscosity of flexible (grey) and stiff (orange) epoxy inks with shear rate. c, Complex viscosity of flexible (grey) and stiff (epoxy) ink measured over time on a rheometer at room temperature. d, Storage and loss moduli of flexible (grey) and stiff (orange) epoxy inks as a function of frequency. e, Photograph of a printed epoxy (flexible) hinge that connects two printed epoxy (stiff) regions. Scale bar $=$ $2 \mathrm{~mm}$. f, Cyclic tests, carried out at a frequency of $0.5 \mathrm{~Hz}$, with bending angles ranging from $18^{\circ}$ to $142^{\circ}$ (sample size $\mathrm{n}=6$ ). The results reveal that the material can sustain $1632 \pm 217$ cycles before failing. (Note: Failure is observed to occur within the flexible epoxy hinge, rather than at the stiff-soft interfaces.) $g$, An image sequence generated from multiple photographs of a Miura fold at various steps of folding. (Expanded side lengths $=64 \mathrm{~mm}$.) 


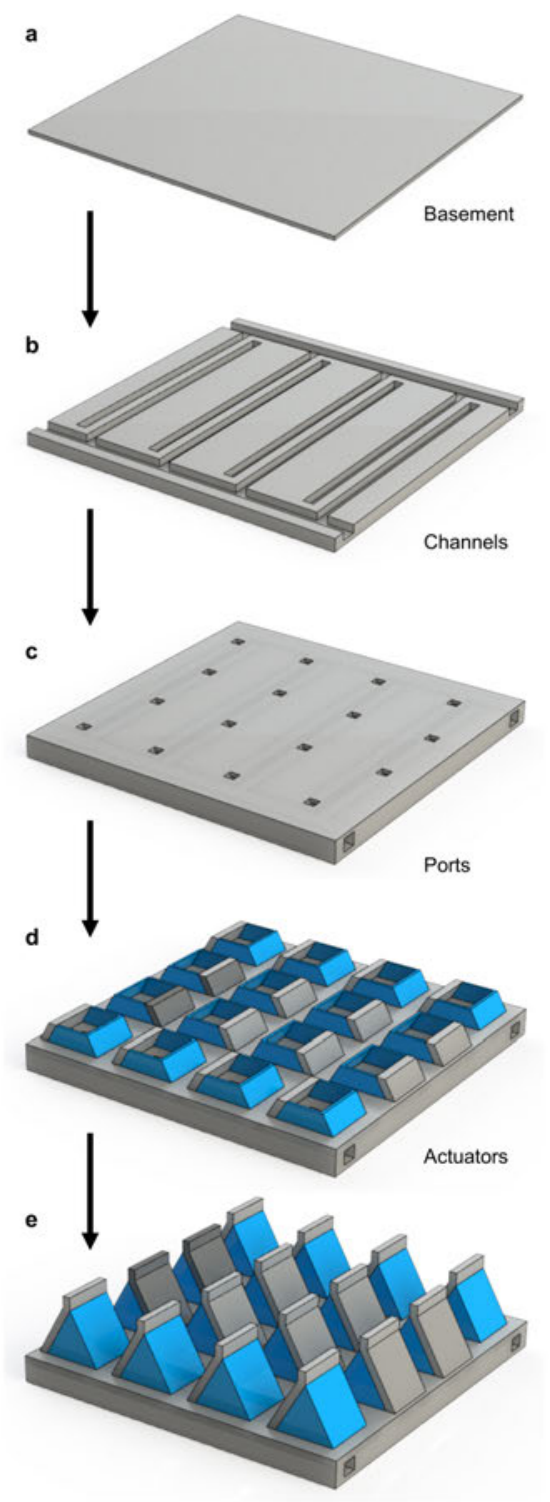

Extended Data Figure 10 | Printing steps used to create soft-robotic millipede walker module. Schematic illustrations of the printing steps: a, An air-tight solid basement (two layers) is printed using the stiff (grey) elastomeric ink. b, A pneumatic channel network is created that connects the vacuum inlets to the actuators (five layers). c, The pneumatic channels are embedded and ports are printed that connect the channels to the actuators (three layers). d, Actuators are printed on top of the ports using stiff (grey) and flexible (blue) silicone inks. e, View of the completed millipede walker module. (Note: Each printed layer is $0.5 \mathrm{~mm}$ in height and the overall dimensions of each actuator unit is $12 \mathrm{~mm} \times 12 \mathrm{~mm} \times 17 \mathrm{~mm}$.) 